Pacific Journal of Mathematics

AN INTERPOLATION THEOREM AND ITS APPLICATIONS TO 


\title{
AN INTERPOLATION THEOREM AND ITS APPLICATIONS TO POSITIVE OPERATORS
}

\author{
V. ToTIK
}

We answer a special case of a problem of Z. Ditzian. The obtained estimate for the Peetre $K$ functional is applied to the characterization of functions for which $\left\|T_{n} f-f\right\|=O\left(n^{-\alpha}\right)(0<\alpha<1)$, where $T_{n}$ is either the Bernstein, Szász-Mirakjan or Baskakov operator or their Kantorovich-invariant and $\|\cdot\|$ denotes either the $L^{p}(p \geq 1)$ or the supremum norm.

1. Let $(a, b)$ be an interval, $B=L^{p}(a, b)(1 \leq p<\infty)$ or $B=$ $C[a, b], \varphi$ a non-negative function on $(a, b)$ and $r \geq 1$ an integer. $Z$. Ditzian [6] estimated the Peetre $K$ functional

$$
K_{r}\left(t^{r}, f\right)=\inf _{g, g^{(r-1)} \text { abs. cont. }}\left(\|f-g\|_{B}+t^{r}\left\|\varphi^{r} g^{(r)}\right\|_{B}\right)
$$

by norms of second order differences of $f$ when $\varphi$ had certain regularity conditions. In connection with this he raised the problem if in the case $(a, b)=(0,1), \quad B=L^{p}(0,1) \quad(1 \leq p<\infty), \quad \varphi(x)=x^{\alpha} \quad(\alpha>0), f \in B$, (support $f) \subseteq(0,3 / 4)$, the estimate

$$
D_{1} \omega_{2 r}^{* *}(f, t) \leq K_{2 r}\left(t^{2 r}, f\right) \leq D_{2} \omega_{2 r}^{* *}(f, t)
$$

holds, where

$$
\begin{aligned}
\omega_{2 r}^{* *}(f, t)= & \sup _{\eta \leq t}\left\{\int_{(r \eta)^{1 /(1-\alpha)}}^{1-C}\left|\Delta_{\eta x^{\alpha}}^{2 r} f(x)\right|^{p} d x\right\}^{1 / p} \\
& +\sup _{\eta \leq t^{1 /(1-\alpha)}}\left\{\int_{r \eta}\left|\Delta_{\eta}^{2 r} f(x)\right|^{p} d x\right\}^{1 / p} \text { for } 0<\alpha<1, \\
\omega_{2 r}^{* *}(f, t)= & \sup _{\eta \leq t}\left\{\int_{(r \eta)^{1 /(1-\alpha)}}^{1-C} \mid \Delta_{\eta x^{\alpha}}^{2 r} f(x) p^{p} d x\right\}^{1 / p} \quad \text { for } \alpha \geq 1,
\end{aligned}
$$

and

$$
\Delta_{h}^{2} f(x)=f(x-h)-2 f(x)+f(x+h) .
$$


In $\$ 2$ we justify this for the special case $r=1$ in a more general setting. Even this has many applications, e.g., the characterization of the function classes

$$
\left\{f \mid\left\|K_{n} f-f\right\|_{L^{p}} \leq K_{f} n^{-\alpha}, n=1,2, \ldots\right\} \quad(0<\alpha<1),
$$

where the $K_{n}$ are the Kantorovich polynomials, as well as other problems concerning Kantorovich type operators. Finally, in the last section we briefly discuss the analogous problem in the $C$-metric.

2. As we have already mentioned, Ditzian's result is complete when $\varphi$ behaves like $x^{\alpha}(\alpha>0)$, however his "modulus of smoothness" is rather complicated and the case of an infinite interval or an infinite singularity at the endpoints is not covered. Ditzian also showed how the general case can be reduced to that where $\varphi$ has no singularity inside $(a, b)$.

We want to estimate

$$
K\left(t^{2}, f\right)=\inf _{\substack{g \\ g^{\prime} \text { abs. cont. }}}\left(\|f-g\|_{L^{p}(a, b)}+t^{2}\left\|\varphi^{2} g^{\prime \prime}\right\|_{L^{p}(a, b)}\right)
$$

by norms of second differences of $f$ which contain the function $\varphi$ itself and not another one of the same order. This will cause several problems, but it turns out to be very fruitful in applications.

Since a linear substitution brings $(a, b)$ to either $(0,1),(0, \infty)$ or $(-\infty, \infty)$, we may suppose $(a, b)$ is $(0,1),(0, \infty)$ or $(-\infty, \infty)$. We also assume $\varphi$ is positive and twice continuously differentiable on $(a, b)$. We need further assumptions on $\varphi$ around the endpoints, which we give for $a=0$ and $b=\infty$ with the agreement that similar conditions hold around $b=1$ or $a=-\infty$. Thus, we suppose:

(1) $\varphi$ is convex or concave in a right ("left") neighbourhood of $a=0$ $(b=\infty)$;

(2) there is a constant $C \geq 2$ such that in these neighbourhoods

$$
(1 / C) \varphi(x) \leq \varphi(y) \leq C \varphi(x) \text { for } x \leq y \leq 2 x,
$$

and

$$
\left|\varphi^{\prime}(x)\right| \leq C \frac{\varphi(x)}{x}, \quad\left|\varphi^{\prime \prime}(x)\right| \leq C \frac{\varphi(x)}{x^{2}}
$$

are satisfied;

(3) furthermore, if $\varphi$ is concave in the mentioned neighbourhood of $a=0$ and $\varphi(0+0) \stackrel{\text { def }}{=} \lim _{x \rightarrow 0+0} \varphi(x)=0$, but $\varphi(x) / x \rightarrow \infty$ as $x \rightarrow 0+$ 0 , then there is a $\gamma<1$ such that $\varphi(x) / x^{\gamma}$ decreases in a neighbourhood of $a=0$. 
For the sake of accuracy we give the analogue of (2) at $b=1$ :

$$
\begin{gathered}
(1 / C) \varphi(x) \leq \varphi(y) \leq C \varphi(x) \quad \text { for }(1-x) \leq(1-y) \leq 2(1-x), \\
\left|\varphi^{\prime}(x)\right| \leq C \frac{\varphi(x)}{1-x}, \quad\left|\varphi^{\prime \prime}(x)\right| \leq C \frac{\varphi(x)}{(1-x)^{2}} .
\end{gathered}
$$

For example our assumptions are satisfied for the functions $x^{\alpha}(\log (1+|x|))^{\beta}, x^{\alpha}|\log x|^{\beta}, x^{\alpha}(1-x)^{\beta}$ provided that at the second one $\alpha \neq 1$ when $\beta>0$ and $a=0$.

Let us agree that $K$ denotes a constant not necessarily the same at each occurrence, but $C, C_{1}, A$ and $A_{p}$ always denote the following constants:

(a) $C$ is as in (2);

(b) $A$ is the constant in

$$
\left\|f^{\prime}\right\|_{L^{p}\left(a^{\prime}, b^{\prime}\right)} \leq A\left(\frac{1}{b^{\prime}-a^{\prime}}\|f\|_{L^{p}\left(a^{\prime}, b^{\prime}\right)}+\left(b^{\prime}-a^{\prime}\right)\left\|f^{\prime \prime}\right\|_{L^{p}\left(a^{\prime}, b^{\prime}\right)}\right),
$$

valid for all $a^{\prime}<b^{\prime}, f, f^{\prime \prime} \in L^{p}\left(a^{\prime}, b^{\prime}\right)$ (see [6, Lemma 2.1]);

(c) $A_{1}=2$ and, for $p>1, A_{p}$ is the constant in the maximal inequality $\left(A_{p} \leq 2(5 p /(p-1))^{p} ;\right.$ see $[14$, p. 7]);

(d) finally, we set

$$
C_{1}=48 C^{6} A A_{p} \text {. }
$$

Let $\psi$ be a twice continuously differentiable function on $(0, \infty)$ with

$$
\psi(x)= \begin{cases}1 & \text { if } x \leq 1 \\ 0 & \text { if } x \geq 2\end{cases}
$$

and $0 \leq \psi(x) \leq 1$ otherwise. We may assume $C$ is so large that $\left|\psi^{\prime}\right| \leq C$ and $\left|\psi^{\prime \prime}\right| \leq C$ are also satisfied.

For small $h>0$ let

$$
\begin{array}{ll}
h^{*}=\inf \{x \mid x-h \varphi(x)>0\} & \text { when }(a, b)=(0,1) \text { or }(a, b)=(0, \infty), \\
h^{*}=\inf \{x \mid x+h \varphi(x)<0\} & \text { when }(a, b)=(-\infty, \infty)
\end{array}
$$

furthermore

$$
\begin{array}{ll}
h^{* *}=\sup \{x \mid x+h \varphi(x)<1\} & \text { when }(a, b)=(0,1), \\
h^{* *}=\sup \{x \mid x-h \varphi(x)>0\} & \text { when }(a, b)=(0, \infty) \text { or }(-\infty, \infty) .
\end{array}
$$

By our assumptions $\varphi(x) / x$ is monotone around the endpoints; hence for small $h, x \pm h \varphi(x) \in(a, b)$ when $x \in\left(h^{*}, h^{* *}\right)$, and, for $(a, b)=(0,1)$ or $(0, \infty),\left(h^{*}, h^{* *}\right)$ is the largest interval with this property. We also have $h \varphi\left(h^{*}\right)=\left|h^{*}\right|$ in every case, $h \varphi\left(h^{* *}\right)=h^{* *}$ when $(a, b)=(0, \infty)$ or 
$(-\infty, \infty)$, and $h \varphi\left(h^{* *}\right)=1-h^{* *}$ when $(a, b)=(0,1)$, provided $h^{*}$ and $h^{* *}$ are finite, respectively.

After these preliminaries we define, for small $t$ (see (2.2)):

$$
\begin{aligned}
\Omega_{0}(f, t) & =\Omega_{0}(t)=\sup _{0<h \leq t}\left\|\Delta_{h \varphi}^{2} f\right\|_{L^{p}\left(\left(C_{1} h\right)^{*},\left(C_{1} h\right)^{* *}\right)}, \\
\Omega_{1}^{(0)}(t) & =\sup _{0<h \leq t^{*}}\left\|\Delta_{h}^{2} f\right\|_{L^{p}\left(h, 2\left(C_{1} h\right)^{*}+h\right)}, \\
\Omega_{1}^{(1)}(t) & =\sup _{0<h \leq t^{*}}\left\|\Delta_{h}^{2} f\right\|_{L^{p}\left(2\left(C_{1} h\right)^{* *}-1-h, 1-h\right)}, \\
\Omega_{2}^{(i)}(t)=\| f-\frac{1}{\left|I_{i}\right|} \int_{I_{i}} f(u) d u & \quad-\frac{12\left(\cdot-\left|I_{i}\right| / 2\right)}{\left|I_{i}\right|^{3}} \int_{I_{i}} f(u)\left(u-\frac{\left|I_{i}\right|}{2}\right) d u \|_{L^{p}\left(I_{i}\right)},
\end{aligned}
$$

where $i=0$ or $1, I_{0}=\left(0,2\left(C_{1} t\right)^{*}\right)$, and $I_{1}=\left(2\left(C_{1} t\right)^{*}-1,1\right)$. Finally with the agreement $\int_{\infty}^{\infty} f=\int_{-\infty}^{-\infty} f=0$, we put

$$
\begin{aligned}
\Omega_{3}^{(\infty)}(t) & =\|f\|_{L^{p}\left(\left(C_{1} t\right)^{* *} / 2, \infty\right)}, \\
\Omega_{3}^{(-\infty)}(t) & =\|f\|_{L^{p}\left(-\infty, 2\left(C_{1} t\right)^{*}\right)}
\end{aligned}
$$

(note that $\Omega_{j}^{(0)}$ and $\Omega_{j}^{(1)}(j=1,2)$ are the same conditions around $a=0$ and $b=1$, respectively).

Setting

$$
\begin{aligned}
& \delta_{0}= \begin{cases}0 & \text { if } \varphi(0+0)=0, \\
1 & \text { if } \varphi(0+0)>0,\end{cases} \\
& \delta_{1}= \begin{cases}0 & \text { if } \varphi(1-0)=0, \\
1 & \text { if } \varphi(1-0)>0\end{cases}
\end{aligned}
$$

and

$$
\delta_{ \pm \infty}=\left\{\begin{array}{lll}
0 & \text { if } \lim _{x \rightarrow \pm \infty}\left|\frac{\varphi(x)}{x}\right|<\infty, \\
1 & \text { if } \lim _{x \rightarrow \pm \infty}\left|\frac{\varphi(x)}{x}\right|=\infty,
\end{array}\right.
$$


we can now define for small $t$, say $0<t \leq t_{0}$, our moduli of smoothness:

$$
\begin{gathered}
\omega(f, t)=\Omega_{0}(t)+\left(1-\delta_{0}\right) \Omega_{1}^{(0)}(t)+\delta_{0} \Omega_{2}^{(0)}(t) \\
+\left(1-\delta_{1}\right) \Omega_{1}^{(1)}(t)+\delta_{1} \Omega_{2}^{(1)}(t) \\
\text { when }(a, b)=(0,1) ; \\
\omega(f, t)=\Omega_{0}(t)+\left(1-\delta_{0}\right) \Omega_{1}^{(0)}(t)+\delta_{0} \Omega_{2}^{(0)}(t)+\delta_{\infty} \Omega_{3}^{(\infty)}(t) \\
\text { when }(a, b)=(0, \infty) ;
\end{gathered}
$$

and

$$
\begin{array}{r}
\omega(f, t)=\Omega_{0}(t)+\delta_{\infty} \Omega_{3}^{(\infty)}(t)+\delta_{-\infty} \Omega_{3}^{(-\infty)}(t) \\
\text { when }(a, b)=(-\infty, \infty) ;
\end{array}
$$

and for these we have

THEOREM 1. Let $\varphi, K(t, f)$ and $\omega$ be as above. There is a constant $K$ independent of $f \in L^{p}(a, b)(1 \leq p<\infty)$ and $0<t \leq t_{0}$ such that

$$
(1 / K) \omega(f, t) \leq K\left(t^{2}, f\right) \leq K \omega(f, t)
$$

holds.

REMARKs. (1) If there is a constant $K>0$ such that for $h^{*} \leq x \leq h^{* *}$ we have $1 \pm h \varphi^{\prime}(x) \geq K$, then we can write

$$
\Omega_{0}^{*}(t)=\sup _{0<h \leq t}\left\|\Delta_{h \varphi}^{2} f\right\|_{L^{p}\left(h^{*}, h^{* *}\right)}
$$

rather than $\Omega_{0}(t)$. Also, in lieu of $\Omega_{1}^{(0)}(t)$ and $\Omega_{1}^{(1)}(t)$ we can always write

$$
\Omega_{1}^{*(0)}(t)=\sup _{0<h \leq t^{*}}\left\|\Delta_{h}^{2} f\right\|_{L^{p}(h, \xi)}
$$

and

$$
\Omega_{1}^{*(1)}(t)=\sup _{0<h \leq t^{*}}\left\|\Delta_{h}^{2} f\right\|_{L^{p}(\xi, 1-h)}
$$

( $\xi \in(0,1)$ is fixed). The estimate of Theorem 1 holds just as well. Both of these statements follow easily from the proof below.

The above remark enables us to write convenient bounds at the norms in the applications, e.g. if $(a, b)=(0,1)$ and $\varphi(x)=\sqrt{x(1-x)}$, then $h^{*}=h^{2} /\left(1+h^{2}\right), h^{* *}=1 /\left(1+h^{2}\right)$, but we shall write

$$
\omega^{*}(f, t)=\sup _{0<h \leq t}\left\|\Delta_{h \varphi}^{2} f\right\|_{L^{p}\left(h^{2}, 1-h^{2}\right)}+\sup _{0<h \leq t^{2}}\left\|\Delta_{h}^{2} f\right\|_{L^{p}(h, 1-h)}
$$


rather than $\omega$, and the estimate

$$
(1 / K) \omega^{*}(f, t) \leq K\left(t^{2}, f\right) \leq K \omega^{*}(f, t)
$$

holds just as well as in Theorem 1.

(2) When $(a, b)=(0,1)$ and $\varphi(x)=x^{\alpha}(\alpha>0)$, then we obtain (1.1) for $r=1$ (see also Remark 1). The case $r>1$ remains open.

(3) $\Omega_{2}^{(i)}(t)$ and $\Omega_{3}^{( \pm \infty)}(t)$ are not true moduli of smoothness. They simply measure the growth of $f$ around the endpoints (for the sake of clarity we remark that in $\Omega_{2}^{(i)}$,

$$
l_{i}(x)=\frac{1}{\left|I_{i}\right|} \int_{I_{i}} f(u) d u-\frac{12\left(x-\left|I_{i}\right| / 2\right)}{\left|I_{i}\right|^{3}} \int_{I_{i}} f(u)\left(u-\frac{\left|I_{i}\right|}{2}\right) d u
$$

is the "orthogonal projection" of $f$ onto the set of the linear functions, i.e. $l_{l}$ is the only linear function for which

$$
\left.\int_{I_{i}} l_{i}(t) d t=\int_{I_{i}} f(t) d t \text { and } \int_{I_{t}} t l_{i}(t) d t=\int_{I_{t}} t f(t) d t\right) .
$$

We mention two corollaries which are by no means trivial.

COROLlaRY 1. If $\varphi_{1}$ and $\varphi_{2}$ are two functions satisfying our conditions and if $\omega_{\varphi_{1}}$ and $\omega_{\varphi_{2}}$ are the corresponding moduli of smoothness, then $\varphi_{1} \leq K \varphi_{2}$ implies

$$
\omega_{\varphi_{1}}(f, t) \leq K_{1} \omega_{\varphi_{2}}(f, t) \quad\left(0<t \leq t_{0}\right)
$$

for all $f \in L^{p}(a, b)$ with $K_{1}$ independent of $f$ and $t$.

Corollary 2. $\omega(f, \lambda t) \leq K \lambda^{2} \omega(f, t)$ for $\lambda \geq 1$ with $K$ independent of $\lambda \geq 1, f \in L^{p}(a, b)$ and $0 \leq t \leq t_{0}$.

Proof of Theorem 1. First we show that $K\left(t^{2}, f\right) \leq K \omega(f, t)$.

(I) The case $(a, b)=(0,1)$. It is enough to prove that for $f \in L^{p}[0,1]$ and (support $f) \subseteq(0,3 / 4)$, we have $K\left(t^{2}, f\right) \leq K \omega(f, t)$ (see [6, p. 310]).

(a) First let us suppose $\varphi(0+0)=0, \varphi$ increases and $\varphi(x) / x$ decreases in the interval $(0, d)$ and $\lim _{x \rightarrow 0+0} \varphi(x) / x=\infty$. In this case $\delta_{0}=0$ and $h^{*}>0$ for all $h>0$ (see (2.4)).

We show the existence of a function $f_{t}$ for which

$$
\begin{gathered}
\left\|f-f_{t}\right\|_{L^{p}\left(\left(C_{1} t\right)^{*}, 7 / 8\right)}+t^{2}\left\|\varphi^{2} f_{t}^{\prime \prime}\right\|_{L^{p}\left(\left(C_{1} t\right)^{*}, 7 / 8\right)} \\
\leq K \omega(f, t)+\frac{12 C^{3} A_{p}}{C_{1}} K\left(t^{2}, f\right)
\end{gathered}
$$

provided $t$ is sufficiently small (here $K$ is independent of $f$ and $t$ ). 
We may suppose $0<t<1 / 8$. For the function

$$
f_{t}(x)=\frac{1}{t^{2}} \int_{0}^{t} d u \int_{0}^{u}(f(x+v \varphi(x))+f(x-v \varphi(x))) d v
$$

we have (see Minkowski's inequality in [14, p. 271])

$$
\left\|f-f_{t}\right\|_{L^{p}\left(\left(C_{1} t\right)^{*}, 7 / 8\right)} \leq \frac{1}{t^{2}} \int_{0}^{t} d u \int_{0}^{u}\left\|\Delta_{v \varphi}^{2} f\right\|_{L^{p}\left(\left(C_{1} v\right)^{*}, 7 / 8\right)} d v \leq \omega(f, t),
$$

and a simple calculation shows that, with $\varphi=\varphi(x)$,

$$
\begin{aligned}
\varphi^{2} f_{t}^{\prime \prime}(x)= & \left(-2 \varphi^{\prime \prime} \varphi+6 \varphi^{\prime 2}\right)\left(f_{t}-f\right) \\
& +\left(\varphi \varphi^{\prime \prime}-4 \varphi^{\prime}\right) \frac{1}{t} \int_{0}^{t} \Delta_{u \varphi}^{2} f(x) d u \\
& +\varphi^{\prime 2} \Delta_{t \varphi}^{2} f(x)+t^{-2} \Delta_{t \varphi}^{2} f(x) \\
& +\frac{2 \varphi^{\prime}}{t^{2}} \int_{0}^{t}[f(x+t \varphi)-f(x-t \varphi) \\
& \quad-2 f(x+u \varphi)+2 f(x-u \varphi)] d u
\end{aligned}
$$

Hence, using the fact that for $\left(C_{1} t\right)^{*} \leq x \leq 7 / 8$ and sufficiently small $t$ we have, by $\varphi(y) \neq O(y)(y \rightarrow 0)$,

$$
\varphi^{\prime 2}+\left|\varphi^{\prime \prime} \varphi\right| \leq \frac{2 C^{2} \varphi^{2}\left(\left(C_{1} t\right)^{*}\right)}{\left(C_{1} t\right)^{* 2}}=\frac{2 C^{2}}{\left(C_{1} t\right)^{2}} \leq \frac{1}{t^{2}},
$$

we obtain from Minkowski's inequality

$$
\begin{aligned}
& t^{2}\left\|\varphi^{2} f_{t}^{\prime \prime}\right\|_{L^{p}\left(\left(C_{1} t\right)^{*}, 7 / 8\right)} \\
& \leq t^{2} t^{-2}\left\{8\left\|f_{t}-f\right\|_{L^{p}\left(\left(C_{1} t\right)^{*}, 7 / 8\right)}+\frac{5}{t} \int_{0}^{t}\left\|\Delta_{u \varphi}^{2}\right\|_{L^{p}\left(\left(C_{1} u\right)^{*}, 7 / 8\right)} d u\right. \\
& \left.\quad+\left\|\Delta_{t \varphi}^{2} f\right\|_{L^{p}\left(\left(C_{1} t\right)^{*}, 7 / 8\right)}\right\} \\
& \quad+\left\|\Delta_{t \varphi}^{2}\right\|_{L^{p}\left(\left(C_{1} t\right)^{*}, 7 / 8\right)}+B(f) \\
& \leq 15 \omega(f, t)+B(f)
\end{aligned}
$$

where

$$
\begin{aligned}
B(f)=2 \| \varphi^{\prime} \int_{0}^{t}(f(x+t \varphi) & -f(x-t \varphi) \\
& -2 f(x+u \varphi)+2 f(x-u \varphi)) d u \|_{L^{p}\left(\left(C_{1} t\right)^{*}, 7 / 8\right)} .
\end{aligned}
$$

Let $g \in L^{p}(0,1)$ be a function the derivative of which is locally absolutely continuous with $\varphi^{2} g^{\prime \prime} \in L^{p}(0,1)$. Clearly,

$$
B(f) \leq B(f-g)+B(g)
$$


and

$$
\begin{aligned}
B(f-g) \leq & 2\left(\sup _{\left(C_{1} t\right)^{*} \leq x \leq 7 / 8}\left|\varphi^{\prime}(x)\right|\right) \\
& \times 6 t \sup _{|u| \leq t}\|(f-g)(\cdot+u \varphi)\|_{L^{p}\left(\left(C_{1} t\right)^{*}, 7 / 8\right)} \\
\leq & 2 \cdot 12 C t \frac{\varphi\left(\left(C_{1} t\right)^{*}\right)}{\left(C_{1} t\right)^{*}}\|f-g\|_{L^{p}} \\
\leq & \frac{24 C}{C_{1}}\|f-g\|_{L^{p}}
\end{aligned}
$$

where we used the fact that for the substitution $\rho=x+u \varphi(x)$ we have

$$
\begin{aligned}
d \rho & =\left(1+u \varphi^{\prime}(x)\right) d x \geq\left(1-t C \frac{\varphi\left(\left(C_{1} t\right)^{*}\right)}{\left(C_{1} t\right)^{*}}\right) d x \\
& =\left(1-\frac{C}{C_{1}}\right) d x \geq \frac{1}{2} d x
\end{aligned}
$$

when $|u| \leq t, \frac{7}{8} \geq x>\left(C_{1} t\right)^{*}$ and $t$ is small enough. Also, for small $t$, $\frac{7}{8} \geq x>\left(C_{1} t\right)^{*}$ and $|v| \leq t \varphi$, we have

$$
\begin{aligned}
x+v \geq & x\left(1-t \frac{\varphi(x)}{x}\right) \geq x\left(1-t \frac{\varphi\left(\left(C_{1} t\right)^{*}\right)}{\left(C_{1} t\right)^{*}}\right) \\
\geq & x\left(1-\frac{t}{C_{1} t}\right) \geq \frac{x}{2}, \\
& x+v \leq x(1+t \varphi(x) / x) \leq 2 x,
\end{aligned}
$$

and, hence, by Taylor's formula,

$$
\begin{aligned}
& \left|\int_{0}^{t}(g(x+t \varphi)-g(x-t \varphi)-2 g(x+u \varphi)+2 g(x-u \varphi)) d u\right| \\
& =\mid \int_{0}^{t} g^{\prime}(x)(2 t \varphi-4 u \varphi) d u \\
& \quad+\int_{0}^{t}\left[\int_{0}^{t \varphi}(t \varphi-v) g^{\prime \prime}(x+v) d v-\int_{0}^{-t \varphi}(-t \varphi-v) g^{\prime \prime}(x+v) d v\right. \\
& \quad-2 \int_{0}^{u \varphi}(u \varphi-v) g^{\prime \prime}(x+v) d v \\
& \left.\quad+2 \int_{0}^{-u \varphi}(-u \varphi-v) g^{\prime \prime}(x+v) d v\right] d u \mid \\
& \leq 6 t^{3} \varphi^{2}\left(\max _{|v| \leq t \varphi} \frac{1}{\varphi^{2}(x+v)}\right) \cdot M\left(\varphi^{2} g^{\prime \prime} ; x\right) \\
& \leq 6 C^{2} t^{3} M\left(\varphi^{2} g^{\prime \prime} ; x\right) \quad\left(\frac{7}{8} \geq x \geq\left(C_{1} t\right)^{*}\right),
\end{aligned}
$$


where $M(g ; x)$ denotes the maximal function of $g$. This and the maximal inequality give, for $1<p<\infty$ and small $t$,

$$
\begin{aligned}
B(g) & \leq 2 \cdot 6 C^{2} t^{3}\left(\max _{\left(C_{1} t\right)^{*} \leq x \leq 7 / 8} \mid \varphi^{\prime}(x) \|\right)\left\|M\left(\varphi^{2} g^{\prime \prime}\right)\right\|_{L^{p}} \\
& \leq 12 C^{3} A_{p} t^{3} \frac{\varphi\left(\left(C_{1} t\right)^{*}\right)}{\left(C_{1} t\right)^{*}}\left\|\varphi^{2} g^{\prime \prime}\right\|_{L^{p}} \\
& \leq 12 C^{3} A_{p} C_{1}^{-1} t^{2}\left\|\varphi^{2} g^{\prime \prime}\right\|_{L^{p}} .
\end{aligned}
$$

For $p=1$,

$$
\begin{aligned}
& \left|\int_{0}^{t} \int_{0}^{ \pm u \varphi}( \pm u \varphi-v) g^{\prime \prime}(x+v) d v d u\right| \\
& \quad \leq t \varphi\left(\max _{|v| \leq t \varphi} \frac{1}{\varphi^{2}(x+v)}\right) \int_{0}^{t}\left|\int_{0}^{ \pm u \varphi} \varphi^{2}(x+v)\right| g^{\prime \prime}(x+v)|d v| d u \\
& \quad \leq C^{2} t \varphi \varphi^{-2} \int_{0}^{t} d u \int_{0}^{u} \varphi^{2}(x \pm \tau \varphi)\left|g^{\prime \prime}(x \pm \tau \varphi)\right| \varphi d \tau \\
& \quad=C^{2} t \int_{0}^{t}(t-\tau) \varphi^{2}(x \pm \tau \varphi)\left|g^{\prime \prime}(x \pm \tau \varphi)\right| d \tau \\
& \quad \leq C^{2} t^{2} \int_{0}^{t} \varphi^{2}(x \pm \tau \varphi)\left|g^{\prime \prime}(x \pm \tau \varphi)\right| d \tau
\end{aligned}
$$

and so, by Taylor's formula,

$$
\begin{aligned}
B(g) \leq & 2 \cdot 6 C^{2} t^{2}{\left.\underset{\left(C_{1} t\right)^{*} \leq x \leq 7 / 8}{\max }\left|\varphi^{\prime}(x)\right|\right)} \times \max _{ \pm}\left\|\int_{0}^{t} \varphi^{2}(x \pm \tau \varphi)\left|g^{\prime \prime}(x \pm \tau \varphi)\right| d \tau\right\|_{L^{\prime}\left(\left(C_{1} t\right)^{*}, 7 / 8\right)} \\
\leq & 12 C^{3} C_{1}^{-1} t \int_{0}^{t} \int_{\left(C_{1} t\right)^{*}}^{7 / 8}\left(\varphi^{2}\left|g^{\prime \prime}\right|\right)(x \pm \tau \varphi(x)) d x d \tau \\
\leq & 24 C^{3} C_{1}^{-1} t^{2}\left\|\varphi^{2} g^{\prime \prime}\right\|_{L^{1}}
\end{aligned}
$$

where at the last step we used (2.8).

By (2.7), (2.9) and (2.10)

$$
B(f) \leq 12 C^{3} A_{p} C_{1}^{-1}\left(\|f-g\|_{L^{p}}+t^{2}\left\|\varphi^{2} g^{\prime \prime}\right\|_{L^{p}}\right) .
$$

Taking on the right side the infimum over all possible $g$, we obtain

$$
B(f) \leq 12 C^{3} A_{p} C_{1}^{-1} K\left(t^{2}, f\right)
$$

for all sufficiently small $t$, and (2.5) has been verified. 
For the function

$$
f_{t}^{*}(x)=\frac{4}{t^{* 2}} \iint_{0}^{t^{*} / 2}(2 f(x+u+v)-f(x+2(u+v))) d u d v,
$$

we have, for small $t$ (see e.g. [6, pp. 319-320]),

$$
\left\|f-f_{t}^{*}\right\|_{L^{p}\left(0,2\left(C_{1} t\right)^{*}\right)} \leq \omega(t) \quad(:=\omega(f, t)),
$$

and since in our case $\varphi(x) / x^{\gamma}$ decreases around $x=0$ for some $\gamma<1$, we also have

$$
\begin{aligned}
t^{2}\left\|\varphi^{2} f_{t}^{* \prime \prime}\right\|_{L^{p}\left(0,2\left(C_{1} t\right)^{*}\right)} & \leq t^{2} \varphi^{2}\left(2\left(C_{1} t\right)^{*}\right)\left\|f_{t}^{* \prime \prime}\right\|_{L^{p}\left(0,2\left(C_{1} t\right)^{*}\right)} \\
& \leq C t^{2} \varphi^{2}\left(\left(C_{1} t\right)^{*}\right)\left(t^{*}\right)^{-2} \omega(t) \\
& =C t^{2}\left(t^{*}\right)^{-2} \omega(t) \varphi^{2}\left(\left(C_{1} t \frac{\varphi\left(\left(C_{1} t\right)^{*}\right)}{\left(\left(C_{1} t\right)^{*}\right)^{\gamma}}\right)^{1 /(1-\gamma)}\right) \\
& \leq C t^{2}\left(t^{*}\right)^{-2} \omega(t) \varphi^{2}\left(\left(C_{1} t \frac{\varphi\left(t^{*}\right)}{\left(t^{*}\right)^{\gamma}}\right)^{1 /(1-\gamma)}\right) \\
& =C t^{2}\left(t^{*}\right)^{-2} \omega(t) \varphi^{2}\left(C_{1}^{1 /(1-\gamma)} t^{*}\right) \\
& \leq K t^{2}\left(t^{*}\right)^{-2} \omega(t) \varphi^{2}\left(t^{*}\right) \leq K \omega(t)
\end{aligned}
$$

hence

$$
\left\|f-f_{t}^{*}\right\|_{L^{p}\left(0,2\left(C_{1} t\right)^{*}\right)}+t^{2}\left\|\varphi^{2} f_{t}^{* \prime \prime}\right\|_{L^{p}\left(0,2\left(C_{1} t\right)^{*}\right)} \leq K \omega(t) .
$$

Now if $\psi$ is the function of (2.3) and

$$
g_{t}(x)=\psi\left(\left(C_{1} t\right)^{*} x\right) f_{t}^{*}(x)+\left(1-\psi\left(\left(C_{1} t\right)^{*} x\right)\right) f_{t}(x)
$$

(let $f_{t}(x)=0$ for $\frac{7}{8} \leq x \leq 1$ ), then from (2.5) and (2.11) we obtain (using $[6$, p. 310])

$$
\left\|f-g_{t}\right\|_{L^{p}(0,1)}+t^{2}\left\|\varphi^{2} g_{t}^{\prime \prime}\right\|_{L^{p}(0,1)} \leq K \omega(f, t)+\frac{12 C^{3} A_{p} 2 A C^{3}}{C_{1}} K\left(t^{2}, f\right),
$$

by which (see (2.2))

$$
K\left(t^{2}, f\right) \leq K \omega(f, t)+\frac{1}{2} K\left(t^{2}, f\right),
$$

and the proof is complete. 
(b) If $\varphi(x) / x$ increases in a right neighbourhood of $x=0$ or if $\varphi(x) / x$ is bounded there, then $h^{*}=0$ for small $h$, and for $f_{t}$ as in (2.5) we have, for small $t$,

$$
\left\|f-f_{t}\right\|_{L^{p}(0,1)}+t^{2}\left\|\varphi^{2} f_{t}^{\prime \prime}\right\|_{L^{p}(0,7 / 8)} \leq K \omega(f, t)
$$

(see the above proof).

Finally, if $\varphi(0+0)>0$ we can also argue as above, except we must use the linear function

$$
\begin{array}{r}
f_{t}^{* *}(x)=\frac{1}{|I|} \int_{I} f(u) d u+\frac{(12 x-6|I|)}{|I|^{3}} \int_{I} f(u)\left(u-\frac{|I|}{2}\right) d u, \\
I=\left(0,2\left(C_{1} t\right)^{*}\right)
\end{array}
$$

rather than $f_{t}^{*}$ (for $f_{t}^{* *}$ our assumption gives

$$
\left.\left\|f-f_{t}^{* *}\right\|_{L^{p}(I)}+\left\|\varphi^{2}\left(f_{t}^{* *}\right)^{\prime \prime}\right\|_{L^{p}(I)}=\left\|f-f_{t}^{* *}\right\|_{L^{p}(I)} \leq \omega(t)\right) .
$$

(II) The case $(a, b)=(0, \infty)$. Here we must show that for $f \in L^{p}(0, \infty)$ and (support $f) \subseteq(1, \infty)$, we have $K\left(t^{2}, f\right) \leq K \omega(f, t)$ (see (I) and [6, p. 310]). If $\varphi(x) / x$ is bounded as $x \rightarrow \infty$ then $h^{* *}=\infty$ for small $h$ and the proof of (I)(a) holds here also (even $f_{t}^{*}$ need not be used). If, moreover, $\varphi(x) / x \rightarrow \infty$ as $x \rightarrow \infty$ then $h^{* *}<\infty$ for all $h$. By the method of (I)(a) it can be proved that

$$
\begin{aligned}
& \left\|f-f_{t}\right\|_{L^{p}\left(1 / 2,\left(C_{1} t\right)^{* *}\right)}+t^{2}\left\|\varphi^{2} f_{t}^{\prime \prime}\right\|_{L^{p}\left(1 / 2,\left(C_{1} t\right)^{* *}\right)} \\
& \leq K \omega(f, t)+6 C^{3} A_{p} C_{1}^{-1} K\left(t^{2}, f\right),
\end{aligned}
$$

and since we also have

$$
\|f\|_{L^{p}\left(\left(C_{1} t\right)^{* *} / 2, \infty\right)} \leq \omega(f, t),
$$

the proof can be completed as above.

The case $(a, b)=(-\infty, \infty)$ can be treated similarly.

We now turn to the proof of $\omega(f, t) \leq K K\left(t^{2}, f\right)$. The estimate of

$$
\Omega_{0}(f, t)=\sup _{0 \leq h \leq t}\left\|\Delta_{h \varphi}^{2} f\right\|_{L^{p}\left(\left(C_{1} h\right)^{*},\left(C_{1} h\right)^{* *}\right)}
$$

is standard: Let $g_{t}$ be chosen so that it satisfies

$$
\left\|f-g_{t}\right\|_{L^{p}(a, b)}+t^{2}\left\|\varphi^{2} g_{t}^{\prime \prime}\right\|_{L^{p}(a, b)} \leq 2 K\left(t^{2}, f\right) .
$$


Considering (2.8), which holds for $\left(C_{1} t\right)^{*} \leq x \leq\left(C_{1} t\right)^{* *}$, we obtain, for $p>1$ from the maximal inequality (see also the considerations above),

$$
\begin{aligned}
& \Omega_{0}(f, t) \leq \Omega_{0}\left(f-g_{t}, t\right)+\Omega_{0}\left(g_{t}, t\right) \\
& \leq 8\left\|f-g_{t}\right\|_{L^{p}(a, b)} \\
&+\sup _{0<h \leq t} \| \int_{-h \varphi / 2}^{h \varphi / 2} \frac{1}{\varphi^{2}(\cdot+u+v)} \varphi^{2}(\cdot+u+v) \\
& \qquad \times\left|g_{t}^{\prime \prime}(\cdot+u+v)\right| d u d v \|_{L^{p}\left(\left(C_{1} h\right)^{*},\left(C_{1} h\right)^{* *}\right)} \\
& \leq 8\left\|f-g_{t}\right\|_{L^{p}(a, b)}+K\left\|\varphi^{-2}(h \varphi)^{2} M\left(\varphi^{2} g_{t}^{\prime \prime} ; \cdot\right)\right\|_{L^{p}(a, b)} \\
& \leq K\left(\left\|f-g_{t}\right\|_{L^{p}(a, b)}+t^{2}\left\|\varphi^{2} g_{t}^{\prime \prime}\right\|_{L^{p}(a, b)}\right) \\
& \leq K K\left(t^{2}, f\right) .
\end{aligned}
$$

For $p=1$ use Fubini's theorem (also compare (I) and [6, pp. 317-318]).

Around the endpoints the smoothness of $f$ is also measured by other terms. We shall estimate these only at $a=0$ or $b=\infty$ since the cases $a=-\infty$ or $b=1$ are similar.

The term

$$
\Omega_{1}^{(0)}(f, t)=\sup _{0<h \leq t^{*}}\left\|\Delta_{h}^{2} f\right\|_{L^{p}\left(h, 2\left(C_{1} h\right)^{*}+h\right)}
$$

occurs when $\varphi$ is concave in a right neighbourhood of $a=0, \varphi(0+0)=0$ and $\lim _{x \rightarrow 0+0} \varphi(x) / x=\infty$. By our assumption in this case, $\varphi(x) / x^{\gamma}$ is decreasing around $a=0$ for some $\gamma<1$. For $x \leq h$,

$$
\begin{aligned}
\int_{x}^{x+h / 2} \frac{u}{\varphi^{2}(u)} d u & =\int_{x}^{x+h / 2} \frac{u^{2 \gamma}}{\varphi^{2}(u)} u^{1-2 \gamma} d u \\
& \leq K \frac{h^{2 \gamma}}{\varphi^{2}(h)} \int_{x}^{x+h / 2} u^{1-2 \gamma} d u \\
& \leq K \frac{h^{2 \gamma}}{\varphi^{2}(h)} h^{2-2 \gamma} \leq K \frac{h^{2}}{\varphi^{2}(h)}
\end{aligned}
$$


so the Hölder inequality gives, for $0<h \leq t^{*}$,

$$
\begin{aligned}
& \left\{\int_{0}^{h}\left|\int_{x}^{x+h / 2}(x-u) g_{t}^{\prime \prime}(u) d u\right|^{p} d x\right\}^{1 / p} \\
& \quad \leq\left\{\int_{0}^{h}\left(\int_{x}^{x+h / 2} \varphi^{2}(u)\left|g_{t}^{\prime \prime}(u)\right| \frac{u}{\varphi^{2}(u)} d u\right)^{p} d x\right\}^{1 / p} \\
& \quad \leq K\left\{\int_{0}^{h}\left(\frac{h}{\varphi(h)}\right)^{2 p / a} \int_{x}^{x+h / 2}\left|\varphi^{2}(u) g_{t}^{\prime \prime}(u)\right|^{p} \frac{u}{\varphi^{2}(u)} d u d x\right\}^{1 / p} \\
& \quad \leq K\left(\frac{h}{\varphi(h)}\right)^{2}\left\{\int_{0}^{3 h / 2}\left(\varphi^{2}(u)\left|g_{t}^{\prime \prime}(u)\right|\right)^{p} d u\right\}^{1 / p} \\
& \quad \leq K \frac{\left(t^{*}\right)^{2}}{\varphi^{2}\left(t^{*}\right)}\left\|\varphi^{2} g_{t}^{\prime \prime}\right\|_{L^{p}(a, b)} \\
& \quad \leq K t^{2}\left\|\varphi^{2} g_{t}^{\prime \prime}\right\|_{L^{p}(a, b)} .
\end{aligned}
$$

This and (2.12) yield

$$
\Omega_{1}^{(0)}(f, t) \leq K K\left(t^{2}, f\right)
$$

exactly as in [6, p. 318].

If $a=0$ and $\varphi(0+0)>0$ then we have to estimate

$$
\Omega_{2}^{(0)}(f, t)=\left\|f-\frac{1}{|I|} \int_{I} f(u) d u-\frac{12(\cdot-|I| / 2)}{|I|^{3}} \int_{I} f(\tau)\left(\tau-\frac{|I|}{2}\right) d \tau\right\|_{L^{p}(I)},
$$

where $I=\left(0,2\left(C_{1} t\right)^{*}\right)$. Let us consider the function $g_{t}$ from (2.12). Since $g_{t}^{\prime \prime} \in L^{1}(I)$, we may assume the continuity of $g_{t}^{\prime}(x)$ at $x=0$, i.e.

$$
g^{\prime}(x)=g^{\prime}(0)+\int_{0}^{x} g^{\prime \prime}(u) d u \text {. }
$$

Using Hardy's inequality ([14, p. 272])

$$
\left\|\frac{1}{x} \int_{0}^{x} h(\tau) d \tau\right\|_{L^{p}(0, \infty)} \leq \frac{p}{p-1}\|h\|_{L^{p}(0, \infty)} \quad(p>1)
$$


and the fact that $\varphi(x) \leq K \varphi(u)$ for $u \leq x \leq|I|$, we obtain, for $p>1$,

$$
\begin{aligned}
& \frac{\varphi^{2}(|I|)}{|I|^{2}}\left\|g_{t}(x)-g_{t}(0)-g_{t}^{\prime}(0) x\right\|_{L^{p}(I)} \\
& \quad \leq K\left\|\frac{1}{x} \int_{0}^{x} \frac{\varphi^{2}(\tau)}{\tau}\left|g_{t}^{\prime}(\tau)-g_{t}^{\prime}(0)\right| d \tau\right\|_{L^{p}(I)} \\
& \quad \leq K\left\|\frac{\varphi^{2}(x)}{x} \int_{0}^{x}\left|g_{t}^{\prime \prime}(u)\right| d u\right\|_{L^{p}(I)} \\
& \quad \leq K\left\|\frac{1}{x} \int_{0}^{x} \varphi^{2}(u)\left|g_{t}^{\prime \prime}(u)\right| d u\right\|_{L^{p}(I)} \leq K\left\|\varphi^{2} g_{t}^{\prime \prime}\right\|_{L^{p}(a, b)} .
\end{aligned}
$$

For $p=1$ we obtain, similarly,

$$
\begin{aligned}
& \frac{\varphi^{2}(|I|)}{|I|^{2}}\left\|g_{t}(x)-g_{t}(0)-g_{t}^{\prime}(0) x\right\|_{L^{\prime}(I)} \\
& \quad \leq \frac{\varphi^{2}(|I|)}{|I|^{2}} \int_{0}^{|I|} \int_{0}^{x} \int_{0}^{y}\left|g^{\prime \prime}(u)\right| d u d y d x \\
& \quad \leq \varphi^{2}(|I|) \int_{0}^{|\eta|}\left|g^{\prime \prime}(u)\right| d u \leq K \int_{0}^{|I|} \varphi^{2}(u)\left|g^{\prime \prime}(u)\right| d u .
\end{aligned}
$$

Now $\varphi^{2}(|I|) /|I|^{2} \geq K t^{-2}$, hence the two previous estimates can be written as

$$
\Omega_{2}^{(0)}\left(g_{t}, t\right) \leq K t^{2}\left\|\varphi^{2} g_{t}^{\prime \prime}\right\|_{L^{p}(a, b)},
$$

so

$$
\begin{aligned}
\Omega_{2}^{(0)}(f, t) & \leq \Omega_{2}^{(0)}\left(f-g_{t}, t\right)+\Omega_{2}^{(0)}\left(g_{t}, t\right) \\
& \leq K\left(\left\|f-g_{t}\right\|_{p}+t^{2}\left\|\varphi^{2} g_{t}^{\prime \prime}\right\|_{p}\right) \leq K K\left(t^{2}, f\right) .
\end{aligned}
$$

Finally, for $b=\infty, \varphi(x) / x \rightarrow \infty(x \rightarrow \infty)$ we have to estimate

$$
\Omega_{3}^{(\infty)}(f, t)=\|f\|_{L^{p}\left(\left(C_{1} t\right)^{* *} / 2, \infty\right)} .
$$

Since $g_{t}, g_{t}^{\prime \prime} \in L^{p}(1, \infty)$, Stein's inequality ([13]) gives $g_{t}^{\prime} \in L^{p}(1, \infty)$. However, $g_{t}^{\prime}$ is uniformly continuous on $(1, \infty)$ (take into account that $\left.\varphi^{2} g_{t}^{\prime \prime} \in L^{p}(1, \infty)\right)$, hence $\lim _{x \rightarrow \infty} g_{t}^{\prime}(x)=0$. Similarly, $\lim _{x \rightarrow \infty} g_{t}(x)=0$ and we obtain, from Hardy's inequality ([14, p. 272])

$$
\left\|\int_{x}^{\infty} h(\tau) d \tau\right\|_{L^{p}(0, \infty)} \leq p\|x h(x)\|_{L^{p}(0, \infty)} \quad(p \geq 1),
$$


that for small $t$,

$$
\begin{aligned}
& \frac{\varphi^{2}\left(\frac{1}{2}\left(C_{1} t\right)^{* *}\right)}{\left(\frac{1}{2}\left(C_{1} t\right)^{* *}\right)^{2}}\left\|g_{t}\right\|_{L^{p}\left(\left(C_{1} t\right)^{* * / 2, \infty)}\right.} \\
& \quad \leq p\left\|\int_{x}^{\infty} \frac{\varphi^{2}(u)}{u^{2}}\left|g_{t}^{\prime}(u)\right| d u\right\|_{L^{p}\left(\left(C_{1} t\right)^{* *} / 2, \infty\right)} \\
& \quad \leq p\left\|\frac{\varphi^{2}(x)}{x} g_{t}^{\prime}(x)\right\|_{L^{p}\left(\left(C_{1} t\right)^{* *} / 2, \infty\right)} \\
& \quad \leq p\left\|\int_{x}^{\infty} \frac{\varphi^{2}(u)}{u}\left|g_{t}^{\prime \prime}(u)\right| d u\right\|_{L^{p}\left(\left(C_{1} t\right)^{* *} / 2, \infty\right)} \\
& \leq p^{2}\left\|\varphi^{2} g_{t}^{\prime \prime}\right\|_{L^{p}(a, b)},
\end{aligned}
$$

where we used the fact that $\varphi$ is convex for sufficiently large $x(\varphi(x) / x \rightarrow$ $\infty$ !), hence $\varphi(x) / x$ increases for large $x$. Since

$$
\varphi^{2}\left(\frac{1}{2}\left(C_{1} t\right)^{* *}\right) /\left(\frac{1}{2}\left(C_{1} t\right)^{* *}\right)^{2} \geq K t^{-2} \quad(K>0),
$$

it follows that

$$
\begin{aligned}
\Omega_{3}^{(\infty)}(f, t) & \leq \Omega_{3}^{(\infty)}\left(f-g_{t}, t\right)+\Omega_{3}^{(\infty)}\left(g_{t}, t\right) \\
& \leq\left\|f-g_{t}\right\|_{L^{p}(a, b)}+K t^{2}\left\|\varphi^{2} g_{t}^{\prime \prime}\right\|_{L^{p}(a, b)} \\
& \leq K K\left(t^{2}, f\right),
\end{aligned}
$$

and the proof is complete.

In applications it will be important to supplement Theorem 1 with an estimate of $K\left(t^{2}, f\right)$ by the second difference $\Delta_{h \varphi}^{2} f$ alone. Keeping the above notation let

$$
v(t)=v(f, t)=\sup _{0 \leq h \leq t}\left\|\Delta_{h \varphi}^{2} f\right\|_{L^{p}\left(h^{*}, h^{* *}\right)},
$$

and for this we can prove

THEOREM 2. With the assumptions of Theorem 1 let $\varphi$ have limit zero at finite endpoints of $(a, b)((a, b)=(0,1)$ or $(0, \infty))$ and let $\varphi(x) / x$ be bounded at infinity $((a, b)=(0, \infty)$ or $(-\infty, \infty))$. Then there is a constant K such that

$$
\frac{1}{K} v(f, t) \leq K\left(t^{2}, f\right) \leq K \int_{0}^{t} \frac{v(f, \tau)}{\tau} d \tau
$$

holds for all $f$ and $0<t \leq t_{0}$.

Specially, $K\left(t^{2}, f\right)=O\left(t^{\alpha}\right)$ and $v(f, t)=O\left(t^{\alpha}\right)$ are equivalent for $\alpha>0$. 
REMARK. Our comment concerning the bounds in the norms in Theorem 1 applies also to Theorem 2.

Proof. By Theorem 1 and Remark 1 we need only prove that

$$
\Omega_{1}^{(0)}(t) \leq K \int_{0}^{t} \frac{v(f, \tau)}{\tau} d \tau
$$

when $f \in L^{p}(0,1)$, (support $\left.f\right) \subseteq\left(0, \frac{3}{4}\right)$ and $t^{*}>0$ for $t>0$ (take into account that by assumption in this case there is a $\gamma<1$ for which $\varphi(x) / x^{\gamma}$ decreases in a right neighbourhood of $x=0$; thus $\varphi^{\prime}(x) \leq$ $\gamma \varphi(x) / x$ there so $1-h \varphi^{\prime}(x) \geq 1-h \gamma \varphi(x) / x \geq 1-\gamma\left(x \geq h^{*}\right)$, and we can apply Remark 1).

Let

$$
K^{*}\left(t^{2}, f\right)=\inf _{g}\left(\|f-g\|_{L^{p}\left(\left(C_{1} t\right)^{*}, 7 / 8\right)}+t^{2}\left\|\varphi^{2} g^{\prime \prime}\right\|_{L^{p}\left(\left(C_{1} t\right)^{*}, 7 / 8\right)}\right)
$$

be the incomplete $K$-functional. By the proof of Theorem 1

$$
K^{*}\left(t^{2}, f\right) \leq K v(f, t)+B(f)
$$

(see (2.6)-(2.7)) and, since

$$
\begin{aligned}
x-t \varphi(x) & \geq\left(C_{1} t\right)^{*}-\frac{1}{C_{1}}\left(C_{1} t\right) \varphi\left(\left(C_{1} t\right)^{*}\right)=\left(1-\frac{1}{C_{1}}\right)\left(C_{1} t\right)^{*} \\
& \geq \frac{1}{2}\left(C_{1} t\right)^{*} \geq\left(C_{1} \frac{t}{2}\right) * \quad\left(x \geq\left(C_{1} t\right)^{*}\right)
\end{aligned}
$$

(the last inequality comes from (2.4)), we obtain, as in the proof of Theorem 1,

$$
B(f) \leq 12 C^{3} A_{p} C_{1}^{-1}\left(\|f-g\|_{L^{p}\left(\left(C_{1} t / 2\right)^{*}, 7 / 8\right)}+t^{2}\left\|\varphi^{2} g^{\prime \prime}\right\|_{L^{p}\left(\left(C_{1} t / 2\right)^{*}, 7 / 8\right)}\right),
$$

and, together with this,

$$
B(f) \leq 48 C^{3} A_{p} C_{1}^{-1} K^{*}\left((t / 2)^{2}, f\right) .
$$

This and (2.14) yield (see also (2.2))

$$
\begin{aligned}
K^{*}\left(t^{2}, f\right) \leq & K v(t)+\frac{1}{2} K^{*}\left(\left(\frac{t}{2}\right)^{2}, f\right) \\
\leq & K\left(v(t)+\frac{1}{2} v\left(\frac{t}{2}\right)+\cdots+\frac{1}{2^{n}} v\left(\frac{t}{2^{n}}\right)\right) \\
& +\frac{1}{2^{n+1}} K^{*}\left(\left(\frac{t}{2^{n+1}}\right)^{2}, f\right) \leq K v(t),
\end{aligned}
$$

where we used the fact that $K^{*}(t, f) \leq\|f\|_{L^{p}}$ for all $t$. 
Now

$$
t^{*}=t \varphi\left(t^{*}\right) \leq t \varphi(x) \text { for } x \geq\left(C_{1} t\right)^{*}
$$

so

$$
\inf _{g}\left(\|f-g\|_{L^{p}\left(\left(C_{1} t\right)^{*}, 7 / 8\right)}+t^{* 2}\left\|g^{\prime \prime}\right\|_{L^{p}\left(\left(C_{1} t\right)^{*}, 7 / 8\right)}\right) \leq K^{*}\left(t^{2}, f\right) \leq K v(t)
$$

is also satisfied and this easily implies

$$
\left\|\Delta_{t^{*}}^{2} f\right\|_{L^{p}\left(\left(C_{1} t\right)^{*}+t^{*}, 7 / 8-t^{*}\right)} \leq K v(t) .
$$

By the assumptions on $\varphi$ there is a $\gamma<1$ such that $\varphi(x) / x^{\gamma}$ decreases around $x=0$, and for this $\gamma$ we have

$$
\begin{aligned}
2\left(t^{*}\right)^{1-\gamma} & =2 t\left(\varphi\left(t^{*}\right) /\left(t^{*}\right)^{\gamma}\right) \geq 2 t\left(\varphi\left((2 t)^{*}\right) /\left((2 t)^{*}\right)^{\gamma}\right) \\
& =\left((2 t)^{*}\right)^{1-\gamma}
\end{aligned}
$$

i.e.

$$
(2 t)^{*} \leq 2^{1 /(1-\gamma)} t^{*}
$$

This, (2.15) and the continuity of the mapping $t \rightarrow t^{*}$ imply there is an $L$ and a $K$ such that

$$
\sup _{h \leq t^{*}}\left\|\Delta_{h}^{2} f\right\|_{L^{p}(L h, 7 / 8-h)} \leq K v(t)
$$

for all $t_{0} \geq t \geq 0$.

Since

$$
\left\|\Delta_{h}^{2} f\right\|_{L^{p}(c, d)} \leq n^{2}\left\|\Delta_{h / n}^{2} f\right\|_{L^{p}(C-h+h / n, d+h-h / n)},
$$

one can easily get from the previous estimate that

$$
\sup _{h \leq t^{*}}\left\|\Delta_{h}^{2} f\right\|_{L^{p}(2 h, 3 / 4)} \leq K v(t)
$$

also holds with $K$ independent of small $t$, say $t \leq t_{0}$.

Now let

$$
w(t)=\sup _{h \leq t^{*}}\left\|\Delta_{h}^{2} f\right\|_{L^{p}(h, 1 / 2)} .
$$

Since

$$
\Delta_{h}^{2} f(x)=\Delta_{h / 2}^{2} f\left(x-\frac{h}{2}\right)+2 \Delta_{h / 2}^{2} f(x)+\Delta_{h / 2}^{2} f\left(x+\frac{h}{2}\right),
$$


it follows readily from (2.16)-(2.17) that

$$
\begin{aligned}
w(t) & \leq w(t / 2)+3 \sup _{h \leq t^{*}}\left\|\Delta_{h / 2}^{2} f\right\|_{L^{p}(h, 3 / 4)} \\
& \leq w(t / 2)+K v(t) .
\end{aligned}
$$

Iterating this and taking into account that $f \in L^{p}(a, b)$ implies $w\left(t / 2^{n}\right)$ $=o(1)(n \rightarrow \infty)$, we obtain

$$
w(t) \leq K \sum_{n=0}^{\infty} v\left(\frac{t}{2^{n}}\right) \leq K \int_{0}^{t} \frac{v(\tau)}{\tau} d \tau
$$

and the proof is over.

3. As a first application let us consider the Kantorovich polynomials

$$
K_{n} f(x)=\sum_{k=0}^{n}\left((n+1) \int_{k /(n+1)}^{(k+1) /(n+1)} f(u) d u\right) p_{n, k}(x) \quad(0 \leq x \leq 1),
$$

where

$$
p_{n, k}(x)=\left(\begin{array}{l}
n \\
k
\end{array}\right) x^{k}(1-x)^{n-k}, \quad 0 \leq k \leq n .
$$

These can be used to approximate a function $f \in L^{p}(0,1)(1 \leq p<\infty)$ in the $L^{p}$-norm and the saturation properties of this approximation were settled by Maier [9, 10] and Riemenschneider [12]. It has been an open problem for some years to characterize those functions $f$ for which $\left\|K_{n} f-f\right\|_{L^{p}}=O\left(n^{-\alpha}\right)(0<\alpha<1)$ (see $\left.[2,3,4,5,7]\right)$. We solved this characterization problem in $[18,19]$ and now we give a somewhat different characterization by the aid of Theorem 1. This new approach can be applied to other operators (see the subsequent sections) and it treats the cases $p=1$ and $p>1$ simultaneously (our earlier method was very different in these two cases, compare [18] and [19]).

Let $\varphi(x)=\sqrt{x(1-x)}$,

$$
\begin{aligned}
D=\left\{g \mid g \in L^{p}(0,1), g^{\prime}\right. \text { absolutely continuous, } \\
\\
\left.x(1-x) g^{\prime \prime}(x) \in L^{p}(0,1)\right\},
\end{aligned}
$$

and

$$
\begin{aligned}
& S g(x)=\left(x(1-x) g^{\prime \prime} \cdot(x), g(x)\right), \\
& \|S g\|_{p}=\left\|\varphi^{2} g^{\prime \prime}\right\|_{L^{p}(0,1)}+\|g\|_{L^{p}(0,1)} .
\end{aligned}
$$

Then $D \subseteq L^{p}(0,1)$ is a linear dense set and $S: D \rightarrow L^{p}(0,1) \times L^{p}(0,1)$ is a linear operator. We set

$$
K\left(t^{2}, f\right)=\inf _{g}\left(\|f-g\|_{p}+t^{2}\left(\|g\|_{p}+\left\|\varphi^{2} g^{\prime \prime}\right\|_{p}\right)\right)
$$


and notice that this $K$ differs from the $K$ functional (2.1) associated with $\varphi$ only by $t^{2}\|g\|_{p}$. Since in (3.1) we may assume $\|g\|_{p} \leq 2\|f\|_{p}$, we obtain that $K\left(t^{2}, f\right)=O\left(t^{2 \alpha}\right)\left(K\right.$ from (3.1)) and $K\left(t^{2}, f\right)=O\left(t^{2 \alpha}\right)$ ( $K$ from (2.1)) are equivalent for $0<\alpha<1$. If we show that

$$
\begin{aligned}
& \left\|K_{n} f-f\right\|_{p} \leq K n^{-1}\|S f\|_{p} \quad(f \in D), \\
& \left\|S K_{n}(f)\right\|_{p} \leq K n\|f\|_{p} \quad\left(f \in L^{p}\right),
\end{aligned}
$$

and

$$
\left\|S K_{n}(f)\right\|_{p} \leq K\|S f\|_{p} \quad(f \in D)
$$

are satisfied with a constant $K$ depending only on $p$, then a result of Grundmann [8] shows that, for $0<\alpha<1,\left\|K_{n} f-f\right\|_{p}=O\left(n^{-\alpha}\right)$ and $K\left(t^{2}, f\right)=O\left(t^{2 \alpha}\right)$ are equivalent. Combining this with Theorem 2 and the following remark, we obtain

THEOREM 3. Let $1 \leq p<\infty, 0<\alpha<1$ and $\varphi(x)=\sqrt{x(1-x)}$. Then for a function $f \in L^{p}(0,1)$ we have

$$
\left\|K_{n} f-f\right\|_{p} \leq K n^{-\alpha} \quad(n=1,2, \ldots)
$$

if and only if

$$
\left\|\Delta_{h \varphi}^{2} f\right\|_{L^{p}\left(h^{2}, 1-h^{2}\right)} \leq K h^{2 \alpha} \quad(h>0) .
$$

Proof of Theorem 3. (3.3) and (3.4) can be proved by a direct calculation (see e.g. $[17,18]$ ), so we justify only (3.2), the strongest of the estimates (3.2)-(3.4).

First we show that

$$
\left\|f^{\prime}\right\|_{p} \leq K\|S f\|_{p} \quad(f \in D) .
$$

Let $f_{1}(x)=f(x) \psi(3 x)$, where $\psi$ is defined in (2.3). Since $f(x)=$ $f(x) \psi(3 x)+f(x)(1-\psi(3 x))$ and $\left\|f^{\prime}\right\|_{L^{p}(1 / 3,2 / 3)} \leq K\|S f\|_{p}$ (see [6, Lemma $2.1])$, by symmetry it is enough to show $\left\|f_{1}^{\prime}\right\|_{L^{p}(0,1 / 3)} \leq K\|S f\|_{p}$. If $h \in$ $L^{q}(0,1)(1 / p+1 / q=1)$ with compact support in $(0,3 / 4)$, then an integration by parts gives

$$
\begin{aligned}
\left|\int_{0}^{1} f_{1}^{\prime}(x) h(x) d x\right| & =\left|\int_{0}^{1} f_{1}^{\prime \prime}(x)\left(\int_{0}^{x} h(\tau) d \tau\right) d x\right| \\
& \leq\left\|\varphi^{2} f_{1}^{\prime \prime}\right\|_{p}\left\|\frac{1}{\varphi^{2}(\cdot)} \int_{0} h(\tau) d \tau\right\|_{q} \\
& \leq K\left\|\varphi^{2} f_{1}^{\prime \prime}\right\|_{p}\|h\|_{q},
\end{aligned}
$$


where at the last step we also used the Hardy inequality (2.13). Since $h \in L^{q}(0,1)$ and (support $\left.h\right) \subseteq(0,3 / 4)$ was arbitrary, (3.7) yields

$$
\begin{aligned}
\left\|f_{1}^{\prime}\right\|_{p} \leq & K\left\|\varphi^{2} f_{1}^{\prime \prime}\right\|_{p} \leq K\left\|\varphi^{2} f^{\prime \prime}\right\|_{L^{p}(0,1 / 3)}+\left(\max \left|\psi^{\prime \prime}\right|\right)\|f\|_{L^{p}(1 / 3,2 / 3)} \\
& +\left(2 \max \left|\psi^{\prime}\right|\right)\left\|f^{\prime}\right\|_{L^{p}(1 / 3,2 / 3)}+\left\|\varphi^{2} f^{\prime \prime}\right\|_{L^{p}(1 / 3,2 / 3)} \\
\leq & K\|S f\|_{p},
\end{aligned}
$$

by which (3.6) is proved.

Now let

$$
B_{n}(f ; x)=B_{n} f(x)=\sum_{k=0}^{n} f\left(\frac{k}{n}\right) p_{n, k}(x)
$$

be the $n$th Bernstein polynomial of $f$. By (3.6) and Jensen's inequality (put $f^{\prime}=0$ outside $\left.[0,1]\right)$,

$$
\begin{aligned}
\| K_{n} f & -B_{n} f \|_{p} \\
& =\left\|\sum_{k=0}^{n}\left((n+1) \int_{k /(n+1)-k / n}^{(k+1) /(n+1)-k / n} \int_{0}^{u} f^{\prime}\left(\frac{k}{n}+v\right) d v d u\right) p_{n, k}(\cdot)\right\|_{p} \\
& \leq\left\|\sum_{k=0}^{n}(n+1) \int_{-1 / n}^{1 / n} O\left(\frac{1}{n}\right)\left|f^{\prime}\left(\frac{k}{n}+v\right)\right| d v p_{n, k}(\cdot)\right\|_{p} \\
& \leq \frac{K}{n}\left\{\int_{0}^{1} \sum_{k=0}^{n}\left((n+1) \int_{-1 / n}^{1 / n}\left|f^{\prime}\left(\frac{k}{n}+v\right)\right|^{p} d v\right) p_{n, k}(x) d x\right\}^{1 / p} \\
& \leq \frac{K}{n}\left\|f^{\prime}\right\|_{p} \leq \frac{K}{n}\|S f\|,
\end{aligned}
$$

where we used the equality

$$
\int_{0}^{1} p_{n, k}(x) d x=\frac{1}{n+1} \quad(1 \leq k \leq n) .
$$

Hence it is enough to prove that for $f \in D$ we have

$$
\left\|B_{n} f-f\right\|_{p} \leq \frac{K}{n}\|S f\|_{p} .
$$

By Taylor's formula

$$
\begin{aligned}
f(t)= & f(x)+f^{\prime}(x)(t-x) \\
& +\frac{1}{2} \int_{0}^{t-x} \frac{t-x-\tau}{(x+\tau)(1-x-\tau)}(x+\tau)(1-x-\tau) g^{\prime \prime}(x+\tau) d \tau .
\end{aligned}
$$


Now for $\tau \in(0, t-x), t, x \in(0,1)$, we have

$$
\left|\frac{t-x-\tau}{(x+\tau)(1-x-\tau)}\right| \leq \frac{|t-x|}{x(1-x)},
$$

so, since $B_{n}(t-x ; x) \equiv 0$, it follows that

$$
\begin{aligned}
& \left|B_{n} f(x)-f(x)\right| \\
& \quad \leq \frac{1}{2} B_{n}\left(\frac{|t-x|}{x(1-x)}\left|\int_{0}^{t-x}(x+\tau)(1-x-\tau)\right| f^{\prime \prime}(x+\tau) \mid d \tau ; x\right) .
\end{aligned}
$$

For $p>1$ we use the maximal function $M(\cdot)$ and the maximal inequality to obtain

$$
\begin{aligned}
\left\|B_{n} f-f\right\|_{p} & \leq \frac{1}{2}\left\|B_{n}\left(\frac{(t-\cdot)^{2}}{\varphi^{2}} M\left(\varphi^{2} f^{\prime \prime} ; \cdot\right) ; \cdot\right)\right\|_{p} \\
& =\frac{K}{n}\left\|M\left(\varphi f^{\prime \prime} ; \cdot\right)\right\|_{p} \leq \frac{K}{n}\left\|\varphi f^{\prime \prime}\right\|_{p} \leq \frac{K}{n}\|S f\|_{p},
\end{aligned}
$$

where we also used the fact that

$$
B_{n}\left((t-x)^{2} ; x\right)=\frac{x(1-x)}{n} \quad(x \in[0,1]) .
$$

For $p=1$ Fubini's theorem yields (put $p_{n,-1} \equiv 0$ )

$$
\begin{aligned}
\| B_{n} f & -f \|_{L^{\prime}(0,1 / 2)} \\
& \leq \frac{1}{2} \int_{0}^{1 / 2}\left\{\sum_{k=0}^{n} \frac{|k / n-x|}{x(1-x)}\left|\int_{x}^{k / n} u(1-u)\right| f^{\prime \prime}(u)|d u| p_{n, k}(x)\right\} d x \\
& \leq \int_{0}^{1}\left\{\sum_{k=0}^{n} \frac{|k / n-x|}{x}\left|\int_{x}^{k / n} u(1-u)\right| f^{\prime \prime}(u)|d u| p_{n, k}(x)\right\} d x \\
& =\int_{0}^{1}\left\{\sum_{k=0}^{n} \frac{k / n-x}{x}\left(\int_{x}^{k / n} u(1-u)\left|f^{\prime \prime}(u)\right| d u\right) p_{n, k}(x)\right\} d x \\
& =\int_{0}^{1}\left\{\sum_{k=0}^{n} \frac{k / n-x}{x}\left(\int_{0}^{k / n} u(1-u)\left|f^{\prime \prime}(u)\right| d u\right) p_{n, k}(x)\right\} d x \\
& =\sum_{k=0}^{n}\left(\int_{0}^{k / n} u(1-u)\left|f^{\prime \prime}(u)\right| d u\right) \int_{0}^{1}\left(p_{n-1, k-1}(x)-p_{n, k}(x)\right) d x \\
& \leq \sum_{k=0}^{n}\left(\int_{0}^{1} u(1-u)\left|f^{\prime \prime}(u)\right| d u\right)\left(\frac{1}{n}-\frac{1}{n+1}\right) \\
& \leq \frac{K}{n}\|S f\|_{L^{1}} .
\end{aligned}
$$

A similar estimate holds on the interval $\left(\frac{1}{2}, 1\right)$ and the proof is complete. 
4. Generalizations. The method of the previous section solves the characterization problem for more "wild" operators than the "regular" Kantorovich ones; furthermore it enables us to give general direct and indirect estimates for the order of Kantorovich approximation (cf. [11]).

First let us consider the operators

$$
K_{n}^{*} f(x)=\sum_{k=0}^{n}\left(\frac{1}{\left|I_{n, k}\right|} \int_{I_{n, k}} f(u) d u\right) p_{n, k}(x) \quad(x \in[0,1]),
$$

where $\left\{I_{n, k}\right\}_{n=1,2, . ; 0 \leq k \leq n}$ is a system of intervals $\subseteq[0,1]$ with centers at the numbers $\left\{x_{n, k}\right\}_{n=1,2, . . ; 0 \leq k \leq n}$. If we assume:

(i) for each $n\left\{x_{n, k}\right\}_{k=0}^{n}$ constitutes an arithmetical sequence (i.e. $\left.x_{n, k+1}=\frac{1}{2}\left(x_{n, k}+x_{n, k+2}\right), k=0,1, \ldots, n-2\right)$, and

(ii) there is a constant $K$ such that for all $n$ and $k$,

$$
\left|x_{n, 0}\right| \leq \frac{K}{n}, \quad\left|1-x_{n, n}\right| \leq \frac{K}{n}, \quad \frac{1}{K n} \leq\left|I_{n, k}\right| \leq \frac{K}{n},
$$

then $K_{n}^{*}$ behaves similarly as $K_{n}$, namely $\left\|K_{n}^{*} f-f\right\|_{L^{p}(0,1)}=O\left(n^{-\alpha}\right)$ is equivalent to (3.5) $\left(f \in L^{p}(0,1), 1 \leq p<\infty, 0<\alpha<1\right)$. An example for $K_{n}^{*}$ is the operator

$$
K_{n}^{*} f(x)=\sum_{k=0}^{n}\left((n+k) \int_{(k+1) /(n+2)-1 / 2(n+k)}^{(k+1) /(n+2)+1 / 2(n+k)} f(u) d u\right) p_{n, k}(x) .
$$

We now turn to the estimate of $\left\|K_{n} f-f\right\|_{L^{p}(0,1)}$.

THEOREM 4. Let $1 \leq p<\infty, f \in L^{p}(0,1), \varphi(x)=\sqrt{x(1-x)}$,

$$
\omega(f, \delta)=\sup _{0<h \leq \delta}\left(\left\|\Delta_{h \varphi}^{2} f\right\|_{L^{p}\left(h^{2}, 1-h^{2}\right)}+\left\|\Delta_{h^{2}}^{2} f\right\|_{L^{p}\left(h^{2}, 1-h^{2}\right)}\right)
$$

and $E_{n}(f)=\left\|K_{n} f-f\right\|_{L^{p}(0,1)}$. Then

(i)

$$
E_{n}(f) \leq K_{p}\left(\omega\left(f ; \frac{1}{\sqrt{n}}\right)+\frac{1}{n}\|f\|_{L^{p}(0,1)}\right)
$$

(ii) for $\gamma>0$,

$$
\omega\left(f, \frac{1}{\sqrt{n}}\right) \leq K_{p, \gamma} \frac{1}{n^{1-\gamma}} \sum_{k=1}^{n} k^{-\gamma} E_{k}(f)+K_{p, \gamma} \frac{1}{n^{1-\gamma}}\|f\|_{p} .
$$

REMARK. If $0<\alpha<1$ and $\alpha+\gamma<1$, then we obtain from (ii) that $E_{n}(f)=O\left(n^{-\alpha}\right)$ implies

$$
\omega\left(f ; \frac{1}{\sqrt{n}}\right) \leq K n^{\gamma-1} \sum_{k=1}^{n} k^{-\gamma-\alpha} \leq K n^{-\alpha} ;
$$


hence Theorem 4 contains Theorem 3 (see Theorems 1 and 2).

Proof. (i) follows easily from the results of the previous sections: with a suitable $g$

$$
\begin{aligned}
E_{n}(f) & \leq E_{n}(f-g)+E_{n}(g) \\
& \leq K\left(\|f-g\|_{p}+\frac{1}{n}\left(\|g\|_{p}+\left\|\varphi^{2} g^{\prime \prime}\right\|_{p}\right)\right) \\
& \leq K\left(K\left(\frac{1}{n}, f\right)+\frac{1}{n}\|f\|_{p}\right) \\
& \leq K\left(\omega\left(f ; \frac{1}{\sqrt{n}}\right)+\frac{1}{n}\|f\|_{p}\right) .
\end{aligned}
$$

In the proof of (ii) let $L$ be a constant for which

$$
\left\|S K_{n} f\right\|_{p} \leq L\|S f\|_{p} \quad(n=1,2, \ldots)
$$

is satisfied for every $n$ and $f \in D$ (see (3.4)), $M=L^{1 / \gamma}$ and let $k_{i}=k_{l, n}$ be defined by

$$
\frac{n}{M^{i+1}}<k_{i} \leq \frac{n}{M^{i}}, \quad E_{k_{i}}(f)=\min _{n / M^{i+1}<k \leq n / M^{i}} E_{k}(f) .
$$

Here $i=0,1, \ldots, i_{n}$, where $i_{n}$ is the first integer with $n / M^{i_{n}+1}<1$. Now by Theorem 1 and (3.3)

$$
\begin{aligned}
\frac{1}{K} \omega\left(f ; \frac{1}{\sqrt{n}}\right) & \leq K\left(\frac{1}{n}, f\right) \leq\left\|f-K_{k_{0}} f\right\|_{p}+\frac{1}{n}\left\|S K_{k_{0}} f\right\|_{p} \\
& \leq E_{k_{0}}(f)+\frac{1}{n}\left\|S K_{k_{0}}\left(f-K_{k_{1}}(f)\right)\right\|_{p}+\frac{1}{n}\left\|S K_{k_{0}} K_{k_{1}} f\right\|_{p} \\
& \leq E_{k_{0}}(f)+\frac{K k_{0}}{n} E_{k_{1}}(f)+\frac{L}{n}\left\|S K_{k_{1}} f\right\|_{p} \leq \ldots \\
& \leq E_{k_{0}}(f)+\frac{K}{n} \sum_{i=0}^{i_{n}-1} L^{i} k_{l} E_{k_{i+1}}(f)+\frac{L^{i_{n}}}{n}\left\|S K_{k_{t_{n}}} f\right\|_{p} \\
& \leq E_{k_{0}}(f)+\frac{K}{n} \sum_{i=0}^{i_{n}-1} \frac{n}{M^{i}} L^{i} E_{k_{i+1}}(f)+\frac{K\|f\|_{p} L^{l_{n}}}{n} \\
& \leq E_{k_{0}}(f)+\frac{K}{n} \sum_{i=0}^{i_{n}-1}\left(\frac{n}{k_{i}}\right)^{\gamma} \frac{n}{M^{i+1}} E_{k_{i+1}}(f)+K \frac{1}{n} M^{i_{n} / \gamma}\|f\|_{p} \\
& \leq \frac{K}{n} \sum_{k=1}^{n}\left(\frac{n}{k}\right)^{\gamma} E_{k}(f)+K n^{\gamma-1}\|f\|_{p}
\end{aligned}
$$

and the proof is complete. 
5. Let us consider the Szász-Mirakjan operators

$$
S_{n} f(x)=\sum_{k=0}^{\infty} f\left(\frac{k}{n}\right) s_{n, k}(x), \quad s_{n, k}(x)=e^{-n x} \frac{(n x)^{k}}{k !}, x \geq 0,
$$

and their Kantorovich variant (see [17])

$$
S_{n}^{*} f(x)=\sum_{k=0}^{\infty}\left(n \int_{k / n}^{(k+1) / n} f(u) d u\right) s_{n, k}(x) .
$$

Let $\varphi(x)=\sqrt{x}$,

$$
D=\left\{f \mid f \in L^{p}(0, \infty), f^{\prime} \text { abs. cont., } x f^{\prime \prime}(x) \in L^{p}(0, \infty)\right\}
$$

and

$$
S f(x)=\left(x f^{\prime \prime}(x), f(x)\right), \quad\|S f\|_{p}=\left\|\varphi^{2} f^{\prime \prime}\right\|_{L^{p}(0, \infty)}+\|f\|_{L^{p}(0, \infty)} .
$$

Exactly as in $\S 3$ it is enough to justify the analogues of (3.2)-(3.4) for the verification of

Theorem 5. Let $\varphi(x)=\sqrt{x}, f \in L^{p}(0, \infty), 1 \leq p<\infty, 0<\alpha<1$. Then

$$
\left\|S_{n}^{*} f-f\right\|_{L^{p}(0, \infty)}=O\left(n^{-\alpha}\right) \quad \text { iff } \quad\left\|\Delta_{h \varphi}^{2} f\right\|_{L^{p}\left(h^{2}, \infty\right)}=O\left(h^{2 \alpha}\right) \quad(h \rightarrow 0) .
$$

Proof. Again we prove only

$$
\left\|S_{n}^{*} f-f\right\|_{p} \leq \frac{K}{n}\|S f\|_{p} \quad(f \in D)
$$

(see also [17]). Using Stein's inequality ([13])

$$
\left\|g^{\prime}\right\|_{L^{p}(0, \infty)} \leq K \sqrt{\|g\|_{p}\left\|g^{\prime \prime}\right\|_{p}} \leq K\left(\|g\|_{L^{p}(0, \infty)}+\left\|g^{\prime \prime}\right\|_{L^{p}(0, \infty)}\right),
$$

the inequality $\left\|f^{\prime}\right\|_{p} \leq K\|S f\|_{p}$ can be shown as the analogue (3.6) in $\S 3$. This reduces (5.1) to

$$
\left\|S_{n} f-f\right\|_{p} \leq \frac{K}{n}\|S f\|_{p} \quad(f \in D)
$$

(see also §3), the proof of which coincides with that of (3.8) when $p>1$.

The case $p=1$, however, requires a finer consideration, which we give below. By the method of $\S 3$ the problem is the estimation of

$$
\int_{0}^{\infty}\left\{\sum_{k=0}^{\infty} \frac{|k / n-x|}{x}\left|\int_{x}^{k / n} u\right| f^{\prime \prime}(u)|d u| s_{n, k}(x)\right\} d x
$$


Here

$$
\left|\frac{k}{n}-x\right|\left|\int_{x}^{k / n} u\right| f^{\prime \prime}(u)|d u|=\left(\frac{k}{n}-x\right) \int_{x}^{k / n} u\left|f^{\prime \prime}(u)\right| d u,
$$

and since the terms under the integral sign are non-negative, we can write (5.2) as

$$
\begin{aligned}
& \sum_{k=0}^{\infty}\left(\int_{0}^{k / n}+\int_{k / n}^{\infty}\right) \frac{k / n-x}{x}\left(\int_{x}^{k / n} u\left|f^{\prime \prime}(u)\right| d u\right) s_{n, k}(x) d x \\
& =\sum_{k=0}^{\infty}\left(\int_{0}^{k / n} u\left|f^{\prime \prime}(u)\right|\left(\int_{0}^{u} \frac{k / n-x}{x} s_{n, k}(x) d x\right) d u\right. \\
& \left.\quad-\int_{k / n}^{\infty} u\left|f^{\prime \prime}(u)\right|\left(\int_{u}^{\infty} \frac{k / n-x}{x} s_{n, k}(x) d x\right) d u\right) \\
& =\int_{0}^{\infty} u\left|f^{\prime \prime}(u)\right| \int_{u}^{\infty} s_{n, 0}(x) d x d u \\
& \quad+\sum_{k=1}^{\infty}\left(\int_{0}^{k / n}+\int_{k / n}^{\infty}\right) u\left|f^{\prime \prime}(u)\right|\left(\int_{0}^{u} \frac{k / n-x}{x} s_{n, k}(x) d x\right) d u
\end{aligned}
$$

where at the last step we used the fact that

$$
\frac{k}{n x} s_{n, k}(x)=s_{n, k-1}(x) \quad(k \geq 1)
$$

and

$$
\int_{0}^{\infty} s_{n, k}(x) d x=\frac{1}{n} \quad(k, n=1,2, \ldots) .
$$

Since $\int_{0}^{u}[(k / n-x) / x] s_{n, k}(x) d x$ increases for $u \leq k / n$ and decreases for $u \geq k / n$, furthermore $\int_{0}^{\infty}[(k / n-x) / x] s_{n, k}(x) d x=0$, we have

$$
\int_{0}^{u} \frac{k / n-x}{x} s_{n, k}(x) d x \geq 0 \quad(u \geq 0) .
$$

Taking into account that, for fixed $u,(k / n)-x$ is non-negative for $x \leq u$ and large enough $k$ and that

$$
\sum_{k=1}^{\infty} \frac{k / n-x}{x} s_{n, k}(x)=-\frac{0-x}{x} s_{n, 0}+S_{n}(t-x ; x)=s_{n, 0}(x),
$$


we can continue the above equality as

$$
\begin{aligned}
\int_{0}^{\infty} u & \left|f^{\prime \prime}(u)\right|\left(\int_{u}^{\infty} s_{n, 0}(x) d x\right) d u \\
& \quad+\int_{0}^{\infty} u\left|f^{\prime \prime}(u)\right| \int_{0}^{u}\left(\sum_{k=1}^{\infty} \frac{k / n-x}{x} s_{n, k}(x)\right) d x d u \\
& =\int_{0}^{\infty} u\left|f^{\prime \prime}(u)\right| \int_{0}^{\infty} s_{n, 0}(x) d x \\
& =\frac{1}{n} \int_{0}^{\infty} u\left|f^{\prime \prime}(u)\right| d u
\end{aligned}
$$

and the proof is complete.

6. Let

$$
\begin{gathered}
V_{n} f(x)=\sum_{k=0}^{\infty} f\left(\frac{k}{n}\right) b_{n, k}(x), \\
b_{n, k}(x)=\left(\begin{array}{c}
n+k-1 \\
k
\end{array}\right) x^{k}(1+x)^{-n-k} \quad(x \geq 0)
\end{gathered}
$$

be the $n$th Baskakov operator and

$$
V_{n}^{*} f(x)=\sum_{k=0}^{\infty}\left(n \int_{k / n}^{(k+1) / n} f(u) d u\right) b_{n, k}(x)
$$

its Kantorovich-variant. For this we have

THEOREM 6. Let $\varphi(x)=\sqrt{x(1+x)}, 1 \leq p<\infty$ and $0<\alpha<1$. For an $f \in L^{p}(0, \infty)$ the statements

$$
\left\|V_{n}^{*} f-f\right\|_{L^{p}(0, \infty)}=O\left(n^{-\alpha}\right)
$$

and

$$
\left\|\Delta_{h \varphi}^{2} f\right\|_{L^{p}\left(2 h^{2}, \infty\right)}=O\left(h^{2 \alpha}\right) \quad(h \rightarrow 0)
$$

are equivalent.

Proof. We follow the arguments of the previous points. Since the analogues of (3.3) and (3.4) for the operators $V_{n}^{*}$ can be proved easily (the computations are very similar to those in $[\mathbf{1 7}, \mathbf{1 8}]$ - see also $[\mathbf{1 6}])$, we need only consider the estimate

$$
\left\|V_{n} f-f\right\|_{L^{p}(0, \infty)} \leq \frac{K}{n}\|S f\|_{p} \quad(f \in D),
$$


where

$$
\begin{gathered}
D=\left\{f \mid f \in L^{p}(0, \infty), f^{\prime} \text { abs. cont., } x(1+x) f^{\prime \prime}(x) \in L^{p}(0, \infty)\right\}, \\
S f(x)=\left(x(1+x) f^{\prime \prime}(x), f(x)\right) \quad(f \in D),
\end{gathered}
$$

and

$$
\|S f\|_{p}=\left\|\varphi^{2} f^{\prime \prime}\right\|_{L^{p}(0, \infty)}+\|f\|_{L^{p}(0, \infty)} .
$$

By Taylor's formula

$$
\begin{aligned}
f(t)= & f(x)+f^{\prime}(x)(t-x) \\
& +\int_{0}^{t-x} \frac{t-x-\tau}{(x+\tau)(1+x+\tau)}(x+\tau)(1+x+\tau) f^{\prime \prime}(x+\tau) d \tau,
\end{aligned}
$$

and here for $\tau \in(0, t-x)$,

$$
\begin{aligned}
& \left|\frac{t-x-\tau}{(x+\tau)(1+x+\tau)}\right| \\
& \quad \leq \begin{cases}4 \frac{|t-x|}{x(1+x)} & \text { for } t \geq \frac{x}{2} \text { or } x \leq 1, \\
2 \frac{|t-x|}{x(1+x)} \frac{(1+x)}{(1+t)} & \text { for } 0 \leq t<\frac{x}{2}, x>1 .\end{cases}
\end{aligned}
$$

Thus,

$$
\begin{aligned}
& \left|V_{n} f(x)-f(x)\right| \\
& \quad \leq K V_{n}\left(\frac{(t-x)^{2}}{x(1+x)} \max \left(1, \frac{1+x}{1+t}\right) ; x\right) M\left(t(1+t) f^{\prime \prime}(t) ; x\right) \\
& \quad \leq \frac{K}{n} M\left(\varphi^{2} f^{\prime \prime} ; x\right),
\end{aligned}
$$

where $M(\cdot)$ is the maximal function, and where we used that $V_{n}(t-x ; x)$ $\equiv 0$ and, by [16, Lemma 4],

$$
V_{n}\left(\frac{(t-x)^{2}}{x(1+x)} \max \left(1, \frac{1+x}{1+t}\right) ; x\right) \leq \frac{K}{n} .
$$


Thus, for $p>1$,

$$
\left\|V_{n} f-f\right\|_{p} \leq \frac{K}{n}\left\|M\left(\varphi^{2} f^{\prime \prime} ; \cdot\right)\right\|_{p} \leq \frac{K}{n}\|S f\|_{p} .
$$

For $p=1$ let

$$
U_{n} f(x)=\sum_{k=0}^{[n x / 2]} f\left(\frac{k}{n}\right) b_{n, k}(x)
$$

By $(6.1)$

$$
\begin{gathered}
\int_{0}^{1}\left|V_{n} f(x)-f(x)\right| d x+\int_{1}^{\infty}\left|V_{n} f(x)-U_{n} f(x)-f(x)\right| d x \\
\leq K \sum_{k=0}^{\infty} \frac{|k / n-x|}{x(1+x)}\left|\int_{x}^{k / n} u(1+u)\right| f^{\prime \prime}(u)|d u| b_{n, k}(x),
\end{gathered}
$$

and the method used in the previous section shows the right-hand side is at most $(K / n)\|S f\|_{1}$.

Thus it remains to prove

$$
\int_{1}^{\infty}\left(\sum_{k=0}^{[n x / 2]}\left|f\left(\frac{k}{n}\right)\right| b_{n, k}(x)\right) d x \leq \frac{K}{n}\|S f\|_{1}
$$

or, by $\left\|f^{\prime}\right\|_{L^{\prime}(0, \infty)} \leq K\|S f\|_{1}($ see $\S 3)$,

$$
\int_{1}^{\infty} \sum_{k=0}^{[n x / 2]}\left(n \int_{k / n}^{(k+1) / n}|f(u)| d u\right) b_{n, k}(x) d x \leq \frac{K}{n}\|S f\|_{1} .
$$

The left-hand side is

$$
\sum_{k=0}^{\infty}\left(n \int_{k / n}^{(k+1) / n}|f(u)| d u\right) \int_{\max (1,2 k / n)}^{\infty} b_{n, k}(x) d x
$$

and if we show that

$$
\int_{\max (1,2 k / n)}^{\infty} b_{n, k}(x) d x \leq \frac{K}{n^{2}} \quad(k=0,1,2, \ldots),
$$

then we obtain the bound

$$
\sum_{k=0}^{\infty} \frac{K}{n} \int_{k / n}^{(k+1) / n}|f(u)| d u=\frac{K}{n}\|f\|_{L^{1}(0, \infty)}
$$

for the left side of (6.2), which already proves (6.2) because $\|f\|_{L^{1}(0, \infty)} \leq$ $\|S f\|_{1}$. 
In (6.3) $x \geq 2 k / n$, hence

$$
\begin{aligned}
b_{n, k}(x)= & (n+k-1)(n-1) x\left(\begin{array}{c}
n+k-2 \\
k
\end{array}\right) x^{k-1}(1+x)^{-n-k} \\
\leq & -3(n+k-1)(k-(n-1) x)\left(\begin{array}{c}
n-1+k-1 \\
k
\end{array}\right) \\
& \times x^{k-1}(1+x)^{-(n-1)-(k+1)} \\
= & -3(n+k+1)\left(b_{n-1, k}(x)\right)^{\prime} \quad(n \geq 3),
\end{aligned}
$$

so, with $\kappa=\max (1,2 k / n)$;

$$
\int_{\kappa}^{\infty} b_{n, k}(x) d x \leq(n+k-1) b_{n-1, k}(\kappa) .
$$

By Stirling's formula we obtain for $k \geq[n / 2]$,

$$
\begin{aligned}
(n+k-1) b_{n-1, k}(\kappa) & \leq \frac{K}{\sqrt{n}} \frac{(n+k)^{n+k-1}}{k^{k} n^{n-2}}\left(\frac{2 k}{n}\right)^{k}\left(1+\frac{2 k}{n}\right)^{-n-k+1} \\
& \leq K \sqrt{n}\left(\frac{2}{(1+k /(n+k))^{(n+k) / k}}\right)^{k} \\
& \leq K \sqrt{n}\left(\frac{2}{(1+(n / 2) /(n+n / 2))^{(n+n / 2) /(n / 2)}}\right)^{k} \\
& \leq K \sqrt{n}\left(\frac{1}{1+1 / 6}\right)^{n / 2} \leq K n^{-2},
\end{aligned}
$$

and, for $0 \leq k<[n / 2]$,

$$
\begin{aligned}
(n+k-1) b_{n-1, k}(\kappa) & =(n+k-1)\left(\begin{array}{c}
n+k-2 \\
k
\end{array}\right) 2^{-n-k+1} \\
& \leq\left(n+\left[\frac{n}{2}\right]-1\right)\left(\begin{array}{c}
n+\left[\frac{n}{2}\right]-2 \\
n
\end{array}\right) 2^{-n-[n / 2]+1} \\
& \leq K n \frac{(1+1 / 2)^{n+n / 2}}{(1 / 2)^{n / 2}} 2^{-n-n / 2} \leq K n\left(\frac{(1+1 / 2)^{3 / 2}}{2}\right)^{n} \\
& \leq K n^{-2},
\end{aligned}
$$

because $(n+k-1) b_{n-1, k}(1)$ increases for $k \leq[n / 2]$.

We have proved (6.3) and, together with this, also Theorem 6. 
7. Finally, we sketch briefly the continuous case. Let $B$ be any of the Banach spaces $C[a, b], C[a, b), C(a, b]$ or $C(a, b)$ with supremum norm \|\| . In the first three cases we can identify $B$ with the subspace of $C(a, b)$ consisting of functions having limit at $a$ and $b$, at $a$, and at $b$, respectively. This enables us to work on $(a, b)$.

We keep the notations and simplifications introduced in $\$ 2$. In the continuous case we need one more assumption on $\varphi$ : if $b=\infty$ and $\lim _{x \rightarrow \infty} \varphi(x) / x=\infty$, then there is a $\gamma>1$ such that $\varphi(x) / x^{\gamma} \nearrow \infty$ in a neighborhood of $b=\infty$ (naturally a similar condition must hold around $-\infty$ when $a=-\infty$ ).

For $f \in B$ let

$$
K\left(t^{2}, f\right)=\inf _{\substack{g \in B \\ g^{\prime} \text { abs. cont. }}}\left(\|f-g\|+t^{2}\left\|\varphi^{2} g^{\prime \prime}\right\|\right) .
$$

We define for small $t$;

$$
\begin{gathered}
\Omega_{0}(t)=\sup _{0<h \leq t}\left\|\Delta_{h \varphi}^{2} f\right\|_{C\left(h^{*}, h^{* *}\right)}, \\
\Omega_{0}^{*}(t)=\sup _{0<h \leq t}\left\|\Delta_{h \varphi}^{2} f\right\|_{C\left(3 h^{*} / 2,2 h^{* *} / 3\right)}, \\
\Omega_{1}^{(i)}(t)=\left\|f-\frac{1}{2\left|I_{i}\right|} \int_{I_{i}} f(u) d u-\frac{12\left(\cdot-\left|I_{i}\right| / 2\right)}{\left|I_{i}\right|^{3}} \int_{I_{i}} f(u)\left(u-\frac{\left|I_{i}\right|}{2}\right) d u\right\|_{C\left(I_{l}\right)},
\end{gathered}
$$

where $i=0$ or $1, I_{0}=\left(0,2 t^{*}\right), I_{1}=\left(2 t^{*}-1,1\right)$,

$$
\Omega_{2}^{(\infty)}(t)=\sup _{x, y \geq t^{* *} / 2}|f(x)-f(y)|
$$

and

$$
\Omega_{2}^{(-\infty)}(t)=\sup _{x, y \leq 2 t^{*}}|f(x)-f(y)|
$$

With

$$
\gamma_{0}=\left\{\begin{array}{ll}
0 & \text { if } \varphi(0+0)<\infty, \\
1 & \text { if } \varphi(0+0)=\infty,
\end{array} \quad \gamma_{1}= \begin{cases}0 & \text { if } \varphi(1-0)<\infty, \\
1 & \text { if } \varphi(1-0)=\infty,\end{cases}\right.
$$

and

$$
\gamma_{ \pm \infty}= \begin{cases}0 & \text { if } \lim _{x \rightarrow \pm \infty} \frac{\varphi(x)}{|x|}<\infty, \\ 1 & \text { if } \lim _{x \rightarrow \pm \infty} \frac{\varphi(x)}{|x|}=\infty\end{cases}
$$


we can define our moduli of smoothness as

$$
\begin{gathered}
\omega(f, t)=\Omega_{0}(t)+\gamma_{0} \Omega_{1}^{(0)}(t)+\gamma_{1} \Omega_{1}^{(1)}(t) \\
\text { when }(a, b)=(0,1) ; \\
\omega(f, t)=\left(1-\gamma_{\infty}\right) \Omega_{0}(t)+\gamma_{\infty} \Omega_{0}^{*}(t)+\gamma_{0} \Omega_{1}^{(0)}(t)+\gamma_{\infty} \Omega_{2}^{(\infty)}(t) \\
\text { when }(a, b)=(0, \infty) ;
\end{gathered}
$$

and

$$
\begin{aligned}
& \omega(f, t)=\left(1-\gamma_{\infty}\right)\left(1-\gamma_{-\infty}\right) \Omega_{0}(t)+\left(\gamma_{\infty}+\gamma_{-\infty}-\gamma_{\infty} \gamma_{-\infty}\right) \Omega_{0}^{*}(t) \\
&+\gamma_{\infty} \Omega_{2}^{(\infty)}(t)+\gamma_{-\infty} \Omega_{2}^{(-\infty)}(t) \quad \\
& \quad \text { when }(a, b)=(-\infty, \infty) ;
\end{aligned}
$$

for these we have

THEOREM 7. Let $B, \varphi, K$ and $\omega$ be as above and $f \in B$. Then there is a constant $K$ independent of $f$ and $t\left(0 \leq t \leq t_{0}\right)$ such that

$$
\frac{1}{K} \omega(f, t) \leq K\left(t^{2}, f\right) \leq K \omega(f, t) .
$$

The proof is similar to that of [6, Theorem 3.1]. We omit the details. Just for the sake of illustration let us prove the estimate $\Omega_{2}^{(\infty)}(t) \leq$ $K K\left(t^{2}, f\right)$. The term $\Omega_{2}^{(\infty)}$ occurs in $\omega$ when $b=\infty$ and $\lim _{x \rightarrow \infty} \varphi(x) / x=$ $\infty$. By our assumption there is a $\gamma>1$ such that $\varphi(x) / x^{\gamma}$ increases for large $x$, say for $x \geq x_{0}$. Let $g_{t}$ be given by

$$
\left\|f-g_{t}\right\|+t^{2}\left\|\varphi^{2} g_{t}^{\prime \prime}\right\| \leq 2 K\left(t^{2}, f\right) .
$$

We have for $\xi>x \geq x_{0}$,

(7.1) $\left|g_{t}^{\prime}(x)-g_{t}^{\prime}(\xi)\right| \leq \int_{x}^{\xi} \frac{u^{2}}{\varphi^{2}(u)}\left|\varphi^{2}(u) g_{t}^{\prime \prime}(u)\right| \frac{1}{u^{2}} d u \leq \frac{x^{2}}{\varphi^{2}(x)}\left\|\varphi^{2} g_{t}^{\prime \prime}\right\| \frac{1}{x}$

so $\varphi^{\prime}$ has a limit at infinity. Since $g_{t}$ is bounded on $(1, \infty)$ we necessarily have $\lim _{\xi \rightarrow \infty} g_{t}^{\prime}(\xi)=0$, by which (see (7.1))

$$
\left|g_{t}^{\prime}(x)\right| \leq 2\left(x / \varphi^{2}(x)\right)\left(K\left(t^{2}, f\right) / t^{2}\right) \quad\left(x \geq x_{0}\right)
$$


Thus, for $y>x \geq \frac{1}{2} t^{*} \geq x_{0}$,

$$
\begin{aligned}
\mid g_{t}(x)- & g_{t}(y)|=| \int_{x}^{y} g_{t}^{\prime}(u) d u \mid \\
& \leq 2 \frac{K\left(t^{2}, f\right)}{t^{2}} \int_{x}^{y} \frac{u^{2 \gamma}}{\varphi^{2}(u)} u^{1-2 \gamma} d u \\
& \leq K \frac{K\left(t^{2}, f\right)}{t^{2}} \frac{x^{2 \gamma}}{\varphi^{2}(x)} \int_{x}^{y} u^{1-2 \gamma} d u \leq K \frac{K\left(t^{2}, f\right)}{t^{2}} \frac{x^{2}}{\varphi^{2}(x)} \\
& \leq K \frac{K\left(t^{2}, f\right)}{t^{2}} \frac{\left(t^{*} / 2\right)^{2}}{\varphi^{2}\left(t^{*} / 2\right)} \leq K \frac{K\left(t^{2}, f\right)}{t^{2}} \frac{\left(t^{*}\right)^{2}}{\varphi^{2}\left(t^{*}\right)} \\
& =K K\left(t^{2}, f\right),
\end{aligned}
$$

so

$$
\begin{aligned}
|f(x)-f(y)| & \leq 2 \| f-g_{t}||+\left|g_{t}(x)-g_{t}(y)\right| \\
& \leq K K\left(t^{2}, f\right) \quad\left(x, y \geq \frac{1}{2} t^{*}\right)
\end{aligned}
$$

as we stated above.

Now let us apply Theorem 6 to the following positive linear operators. Let $T_{n}$ be any of the operators:

$$
\begin{aligned}
& B_{n} f(x)=\sum_{k=0}^{n} f\left(\frac{k}{n}\right)\left(\begin{array}{l}
n \\
k
\end{array}\right) x^{k}(1-x)^{n-k}, \quad f \in C[0,1], \\
& S_{n} f(x)=\sum_{k=0}^{\infty} f\left(\frac{k}{n}\right) e^{-n x} \frac{(n x)^{k}}{k !}, \quad f \in C[0, \infty), \\
& V_{n} f(x)=\sum_{k=0}^{\infty} f\left(\frac{k}{n}\right)\left(\begin{array}{c}
n+k-1 \\
k
\end{array}\right) x^{k}(1+x)^{-n-k}, \quad f \in C[0, \infty),
\end{aligned}
$$

i.e. any of the Bernstein, Szász-Mirakjan and Baskakov operators (see [1, $15,16])$. We put

$$
\begin{gathered}
\omega_{T}(f, \delta)=\sup _{0<h \leq \delta}\left\|\Delta_{h \varphi}^{2} f\right\|_{C\left(h^{2}, 1-h^{2}\right)}, \quad \varphi(x)=\sqrt{x(1-x)}, T_{n}=B_{n}, \\
\omega_{T}(f, \delta)=\sup _{0<h \leq \delta}\left\|\Delta_{h \varphi}^{2} f\right\|_{C\left(h^{2}, \infty\right)}, \quad \varphi(x)=\sqrt{x}, T_{n}=S_{n},
\end{gathered}
$$

and

$$
\omega_{T}(f, \delta)=\sup _{0<h \leq \delta}\left\|\Delta_{h \varphi}^{2} f\right\|_{C\left(2 h^{2}, \infty\right)}, \quad \varphi(x)=\sqrt{x(1+x)}, T_{n}=V_{n},
$$


respectively, and for these we have

THEOREM 8. Let $T_{n}$ and $\omega=\omega_{T}$ be as above and $E_{n}(f)=E_{n}^{T}(f)=$ $\left\|f-T_{n} f\right\|$. Then

(i)

$$
E_{n}(f) \leq K \omega(f, 1 / \sqrt{n})
$$

(ii) for $\gamma>0$,

$$
\omega\left(f, \frac{1}{\sqrt{n}}\right) \leq K_{\gamma} n^{-(1-\gamma)} \sum_{k=0}^{n} k^{-\gamma} E_{k}(f) .
$$

Corollary 3. $\left\|T_{n} f-f\right\|=o(1)$ if and only if $\omega(f, \delta)=o(1)(\delta \rightarrow 0)$.

COROllary 4. For $0<\alpha<1,\left\|T_{n} f-f\right\|=O\left(n^{-\alpha}\right)(n \rightarrow \infty)$ if and only if $\omega(f, \delta)=O\left(\delta^{2 \alpha}\right)(\delta \rightarrow 0)$.

Remarks. (1) When $T_{n}=B_{n}$ Corollary 3 is simply the statement $\left\|B_{n} f-f\right\|_{C[0,1]}=o(1)$ for every $f \in C[0,1]$. However in the cases $T_{n}=S_{n}$ or $T_{n}=V_{n}$, Corollary 3 characterizes those bounded functions for which $T_{n} f(x)-f(x)=o(1)$ uniformly on the positive real line (see also [15, 16]).

(2) For $T_{n}=B_{n}$ Corollary 4 was also proved by Ditzian [6].

(3) Since, e.g.,

$$
\sup _{0<h \leq \delta}\left\|\Delta_{h \varphi}^{2} f\right\|_{C\left(h^{2}, \infty\right)} \leq K h^{2 \alpha}, \quad \varphi(x)=\sqrt{x},
$$

is equivalent to

$$
\varphi^{2 \alpha}(x)\left|\Delta_{h}^{2}(f, x)\right| \leq K h^{2 \alpha} \quad(x \geq h),
$$

we obtain that $S_{n} f-f=O\left(n^{-\alpha}\right)$ is equivalent to

$$
x^{\alpha}|f(x-h)-2 f(x)+f(x+h)| \leq K h^{2 \alpha} \quad(x \geq h)
$$

(cf. [15]).

(4) The Meyer-König and Zeller operators (see [16]) could be treated similarly. 
The proof of Theorem 8 uses Theorem 7 and the arguments applied in $\S 4$, namely if we put

$$
\begin{gathered}
D=\left\{f \mid f \in C[0,1], f^{\prime} \text { abs. cont., }\left|x(1-x) f^{\prime \prime}(x)\right| \leq K\right\}, \\
S f(x)=x(1-x) f^{\prime \prime}(x) \quad\left(T_{n}=B_{n}\right), \\
D=\left\{f \mid f \in C[0, \infty), f^{\prime} \text { abs. cont., }\left|x f^{\prime \prime}(x)\right| \leq K\right\}, \\
S f(x)=x f^{\prime \prime}(x) \quad\left(T_{n}=S_{n}\right),
\end{gathered}
$$

and

$$
\begin{gathered}
D=\left\{f \mid f \in C[0, \infty), f^{\prime} \text { abs. cont., }\left|x(1+x) f^{\prime \prime}(x)\right| \leq K\right\}, \\
S f(x)=x(1+x) f^{\prime \prime}(x) \quad\left(T_{n}=V_{n}\right),
\end{gathered}
$$

respectively, then the estimates

$$
\left\|T_{n} f\right\| \leq K\|f\|, \quad\left\|S T_{n} f\right\| \leq K n\|f\| \quad(f \in B)
$$

and

$$
\left\|T_{n} f-f\right\| \leq \frac{K}{n}\|S f\|, \quad\left\|S T_{n} f\right\| \leq K\|S f\| \quad(f \in D)
$$

are satisfied in every case. We omit the details.

\section{REFERENCES}

[1] M. Becker, Global approximation theorems for Szász-Mirakjan and Baskakov operators in polynomial weight spaces, Indiana Univ. Math. J., 27 (1978), 127-142.

[2] M. Becker, K. J. Lautner, R. J. Nessel and G. J. Worms, On global approximation by Kantorovitch polynomials in $L^{p}$, to appear in: "Constructive Function Theory" (Proc. Conf. Varna, 1981).

[3] M. Becker and R. J. Nessel, On the Global Approximation by Kantorovich Polynomials, in: "Approximation Theory III" (Proc. Conf. Austin, 1979), Academic Press, New York, 1980, 207-212.

, On Global Saturation for Kantorovitch Polynomials, in: “Approximation and Function Spaces" (Proc. Conf., Gdansk, 1979), North-Holland, Amsterdam and Polish Sci. Publ., Warsaw, 1981, 89-101.

[5] _ Some global direct estimates for Kantorovitch polynomials, Analysis, to appear.

[6] Z. Ditzian, On interpolation of $L_{p}[a, b]$ and weighted Sobolev spaces, Pacific J. Math., 90 (1980), 307-323.

[7] Z. Ditzian and C. P. May, $L_{p}$ saturation and inverse theorems for modified Bernstein Polynomials, Indiana Math. J., (1976), 733-751.

[8] A. Grundmann, Inverse Theorems for Kantorovich-Polynomials, in: "Fourier Analysis and Approximation Theory" (Proc. Conf., Budapest, 1976), North-Holland, Amsterdam 1978, 395-401.

[9] V. Maier, The $L_{1}$ saturation class of the Kantorovich operator, J. Approx. Theory, 22 (1978), 223-232.

[10] _ , L L -approximation by Kantorovic operators, Anal. Math., 4 (1978), 289-295. 
[11] M. W. Müller, Die Güte der $L_{p}$-Approximation durch Kantorvic-Polynome, Math. Z., 152 (1976), 243-247.

[12] S. D. Riemenschneider, The $L_{p}$-saturation of the Kantorovic-Bernstein polynomials, J. Approx. Theory, 23 (1978), 158-162.

[13] E. M. Stein, Functions of exponential type, Ann. Math., 65 (1957), 582-592.

[14] _ Singular Integrals, Princeton, New Jersey, 1970.

[15] V. Totik, Uniform approximation by Szász-Mirakjan type operators, Acta Math. Acad. Sci. Hung., 41 (1983/3-4).

[16] __. Uniform approximation by Baskakov and Meyer-König and Zeller operators, Periodica Math., to appear.

[17] _ Approximation by Szász-Mirakjan-Kantorovich operators in $L^{p}(p>1)$, Anal. Math., 9 (1983), 147-167.

[18] — $L^{p}(p>1)$-approximation by Kantorovich polynomials, Analysis, to appear.

[19] __ Approximation in $L^{1}$ by Kantorovich polynomials, Acta Sci. Math., to appear.

[20] , Problems and solutions concerning Kantorovich operators, J. Approx. Theory, 47 (1983), 51-68.

Received July 21, 1982.

BOLYAI INSTITUTE

JÓZSEF ATTILA TUdOMÁNYEgYeTEM, TTK

SZEged, ARAdi V. TERE 1

H-6720, HUNGARY 



\title{
PACIFIC JOURNAL OF MATHEMATICS EDITORS
}

\author{
Donald BABBITT (Managing Editor) \\ University of California \\ Los Angeles, CA 90024 \\ Hugo RossI \\ University of Utah \\ Salt Lake City, UT 84112 \\ C. C. MOore and Arthur Ogus \\ University of California \\ Berkeley, CA 94720
}

J. DUGUNDJI
Department of Mathematics

University of Southern California

Los Angeles, CA 90089-1113

R. FinN and H. SAMELSON

Stanford University

Stanford, CA 94305

\section{ASSOCIATE EDITORS}
R. ARENS
E. F. BECKENBACH
B. H. NeumanN
F. WOLF
K. YosHIDA (1906-1982)

\section{SUPPORTING INSTITUTIONS}

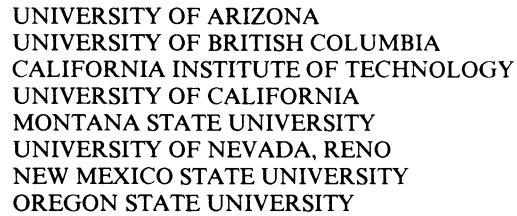

UNIVERSITY OF ARIZONA

UNIVERSITY OF BRITISH COLUMBIA

CALIFORNIA INSTITUTE OF TECHNOLOGY

UNIVERSITY OF CALIFORNIA

MONTANA STATE UNIVERSITY

UNIVERSITY OF NEVADA, RENO

NEW MEXICO STATE UNIVERSITY

OREGON STATE UNIVERSITY

\author{
UNIVERSITY OF OREGON \\ UNIVERSITY OF SOUTHERN CALIFORNIA \\ STANFORD UNIVERSITY \\ UNIVERSITY OF HAWAII \\ UNIVERSITY OF TOKYO \\ UNIVERSITY OF UTAH \\ WASHINGTON STATE UNIVERSITY \\ UNIVERSITY OF WASHINGTON
}

The Supporting Institutions listed above contribute to the cost of publication of this Journal, but they are not owners or publishers and have no responsibility for its content or policies.

Mathematical papers intended for publication in the Pacific Journal of Mathematics should be in typed form or offset-reproduced (not dittoed), double spaced with large margins. Please do not use built up fractions in the text of the manuscript. However, you may use them in the displayed equations. Underline Greek letters in red, German in green, and script in blue. The first paragraph must be capable of being used separately as a synopsis of the entire paper. In particular it should contain no bibliographic references. Please propose a heading for the odd numbered pages of less than 35 characters. Manuscripts, in triplicate, may be sent to any one of the editors. Please classify according to the scheme of Math. Reviews, Index to Vol. 39. Supply name and address of author to whom proofs should be sent. All other communications should be addressed to the managing editor, or Elaine Barth, University of California, Los Angeles, California 90024.

There are page-charges associated with articles appearing in the Pacific Journal of Mathematics. These charges are expected to be paid by the author's University, Government Agency or Company. If the author or authors do not have access to such Institutional support these charges are waived. Single authors will receive 50 free reprints; joint authors will receive a total of 100 free reprints. Additional copies may be obtained at cost in multiples of 50 .

The Pacific Journal of Mathematics is issued monthly as of January 1966. Regular subscription rate: $\$ 132.00$ a year (6 Vol., 12 issues). Special rate: $\$ 66.00$ a year to individual members of supporting institutions.

Subscriptions, orders for numbers issued in the last three calendar years, and changes of address should be sent to Pacific Journal of Mathematics, P.O. Box 969, Carmel Valley, CA 93924, U.S.A. Old back numbers obtainable from Kraus Periodicals Co., Route 100, Millwood, NY 10546.

The Pacific Journal of Mathematics ISSN 0030-8730 is published monthly by the Pacific Journal of Mathematics at P.O. Box 969, Carmel Valley, CA 93924. Application to mail at Second-class postage rates is pending at Carmel Valley, California, and additional mailing offices. Postmaster: Send address changes to Pacific Journal of Mathematics, P. O. Box 969, Carmel Valley, CA 93924.

PUBLISHED BY PACIFIC JOURNAL OF MATHEMATICS, A NON-PROFIT CORPORATION 


\section{Pacific Journal of Mathematics}

\section{Vol. 111, No. $2 \quad$ December, 1984}

Berndt Brenken, Representations and automorphisms of the irrational

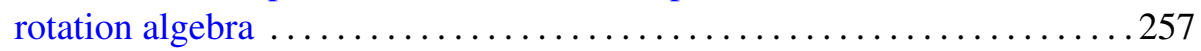

Harold George Diamond, A number theoretic series of I. Kasara . . . . . . 283

Rolf Farnsteiner, On the structure of simple-semiabelian Lie algebras . . . . 287

Guillermo Grabinsky, Poisson process over $\sigma$-finite Markov chains . ......301

Derbiau Frank Hsu and A. Donald Keedwell, Generalized complete

mappings, neofields, sequenceable groups and block designs. I . . . . . 317

William H. Julian and Fred Richman, A uniformly continuous function on

$[0,1]$ that is everywhere different from its infimum $\ldots \ldots \ldots \ldots . \ldots 33$

D. H. Lehmer and Emma Lehmer, The sextic period polynomial .........341

E. Maluta, Uniformly normal structure and related coefficients ...........357

Coy Lewis May, The species of bordered Klein surfaces with maximal

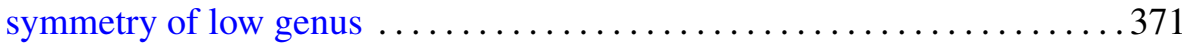

Louis Jackson Ratliff, Jr., On asymptotic prime divisors . . . . . . . . . . . 395

Norbert Riedel, Disintegration of KMS-states and reduction of standard von

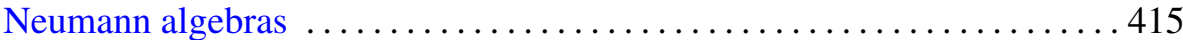

Richard Gordon Swan, $n$-generator ideals in Prüfer domains ...........433

Vilmos Totik, An interpolation theorem and its applications to positive

operators .................................. 447

Richard Vrem, Hypergroup joins and their dual objects 